\title{
Risk factors for early-onset colorectal cancer: a population-based case-control study in Ontario, Canada
}

\author{
Vicky C. Chang ${ }^{1,2}\left(\right.$ D $\cdot$ Michelle Cotterchio ${ }^{1,2} \cdot$ Prithwish De $^{1} \cdot$ Jill Tinmouth $^{1,3,4}$
}

Received: 31 December 2020 / Accepted: 30 May 2021 / Published online: 13 June 2021

(c) The Author(s) 2021

\begin{abstract}
Purpose There has been an alarming increase in colorectal cancer (CRC) incidence among young adults aged $<50$ years, and factors driving this upward trend are unknown. This study investigated associations between various medical, lifestyle, and dietary factors and risk of early-onset CRC (EO-CRC).

Methods A population-based case-control study was conducted in Ontario, Canada during 2018-2019. EO-CRC cases aged 20-49 years $(n=175)$ were identified from the Ontario Cancer Registry; sex- and age group-matched controls $(n=253)$ were recruited through random digit dialing. Data on potential a priori risk factors were collected using a web-based self-reported questionnaire. Odds ratios (OR) and 95\% confidence intervals (CI) were estimated using multivariable logistic regression. Results Family history of CRC in a first- or second-degree relative (OR 2.37; 95\% CI 1.47-3.84), longer sedentary time ( $\geq 10$ vs. $<5$ h/day, OR 1.93; 95\% CI 1.02-3.65), greater consumption of sugary drinks ( $\geq 7$ vs. $<1$ drinks/week, OR 2.99; $95 \%$ CI 1.57-5.68), and a more Westernized dietary pattern (quartile 4 vs. 1, OR 1.92; 95\% CI 1.01-3.66) were each associated with an increased risk of EO-CRC. Conversely, calcium supplement use (OR 0.53; 95\% CI 0.31-0.92), history of allergy or asthma (OR 0.62 ; 95\% CI 0.39-0.98), and greater parity in females ( $\geq 3$ vs. nulliparity, OR $0.29 ; 95 \%$ CI $0.11-0.76$ ) were each associated with a reduced risk.

Conclusion Modifiable factors, particularly sedentary behavior and unhealthy diet including sugary drink consumption, may be associated with EO-CRC risk. Our findings, if replicated, may help inform prevention strategies targeted at younger persons.
\end{abstract}

Keywords Early-onset colorectal cancer $\cdot$ Risk factors $\cdot$ Diet $\cdot$ Sugary drinks $\cdot$ Sedentary behavior

\section{Introduction}

Colorectal cancer (CRC) is the third most commonly diagnosed cancer and the second leading cause of cancer deaths globally [1]. In contrast to declining rates among persons aged 50 years or older, which have been largely attributed

Vicky C. Chang

vickycd.chang@mail.utoronto.ca

1 Prevention and Cancer Control, Clinical Institutes and Quality Programs, Ontario Health (Cancer Care Ontario), Toronto, ON, Canada

2 Dalla Lana School of Public Health, University of Toronto, Toronto, ON, Canada

3 Department of Medicine, Sunnybrook Health Sciences Centre and University of Toronto, Toronto, ON, Canada

4 Institute of Health Policy, Management and Evaluation, University of Toronto, Toronto, ON, Canada to screening, CRC incidence rates among young adults (aged $<50$ ) have increased markedly over the past few decades worldwide [2] and in many countries/regions, including Canada [3], the United States [4], Australia [5], and parts of Europe [6, 7] and Eastern Asia [8]. Reasons underlying the alarming rise in early-onset CRC (EO-CRC) are unknown. The increasing prevalence of several traditional CRC risk factors in younger birth cohorts, such as physical inactivity, obesity, and diabetes, has been hypothesized to contribute to the upward trend [9-11]; however, their associations with EO-CRC risk are not consistently supported by the limited epidemiological evidence to date [12].

EO-CRC occurs more commonly in the distal colon and rectum and are often characterized by more advanced stage at diagnosis and aggressive tumor histology [13]. Despite extensive knowledge regarding the etiology of overall CRC (based primarily on older-onset CRC) [14], there is a paucity of literature on risk factors specific to EO-CRC. A recent 
systematic review and meta-analysis of EO-CRC risk factors identified 20 relevant studies published up to August 2020 and reported significant associations for first-degree family history of CRC, hyperlipidemia, obesity, and alcohol consumption, although analyses for most factors were based on a small number of studies with considerable heterogeneity [15]. One of the first studies investigating EO-CRC risk factors among both men and women was a European hospital-based case-control study conducted in 1985-2009, which reported increased risk associated with family history of CRC, alcohol, and processed meat intake, and no association with physical activity, overweight/obesity, or diabetes [16]. More recently, analyses of prospective cohort data from the Nurses' Health Study revealed an association between obesity [17], as well as sedentary behavior (assessed as TV viewing time) [18], and increased risk of EO-CRC. In addition, relying on administrative or electronic health record (EHR) data, several retrospective cohort [19-22] and case-control [23, 24] studies have been published recently (since 2019) on EO-CRC risk factors. While most of these studies reported increased EO-CRC risk associated with family history of CRC [19-23], personal history of inflammatory bowel disease (IBD) [19, 21, 23], and/or diabetes $[21,22]$, they lacked detailed data on modifiable lifestyle factors, such as diet, and potential confounders.

Beyond conventional CRC risk factors, emerging hypotheses suggest the need to also consider "novel" exposures that may be more prevalent among younger generations [11, 12, 25]. These include Westernized diets, processed foods and additives (e.g., high-fructose corn syrup), modulators of the gut microbiome and/or immune system (e.g., antibiotics, allergies), and radiation from medical procedures (e.g., computed tomography [CT] scans) $[11,12,25,26]$, which have yet to be evaluated in epidemiologic studies in relation to EO-CRC risk. Given the increase in EO-CRC incidence and scarcity of knowledge on risk factors for this largely preventable disease, we conducted a study to evaluate associations of various medical, lifestyle, and dietary factors with EO-CRC risk, covering both previously established risk factors for overall CRC as well as a priori hypothesized novel factors.

\section{Methods}

A population-based case-control study was conducted in Ontario, Canada to investigate a wide range of potential risk factors for EO-CRC. The study protocol was approved by the University of Toronto Health Sciences Research Ethics Board.

\section{Case ascertainment and recruitment}

EO-CRC cases were identified through the Ontario Cancer Registry (OCR), a population-based database of all cancer cases in the province of Ontario. Eligible cases were Ontario residents aged 20-49 years at the time of diagnosis with a pathologically confirmed incident invasive colorectal adenocarcinoma (International Classification of Diseases for Oncology, Third Edition [ICD-O-3] topography codes: C18.0, C18.2-C18.9, C19.9, C20.9) between January 2018 and May 2019. Information on tumor microsatellite instability (MSI) status was obtained from pathology reports. MSI status was determined based on immunohistochemistry staining results for DNA mismatch repair proteins (MLH1, MSH2, MSH6, and PMS2), with cases classified as "MSI-high" if they had abnormal staining for any of the four proteins.

Of the 782 eligible EO-CRC cases identified from the OCR, 220 provided opt-in consent to be contacted by our study (by mailing back a signed consent form to Cancer Care Ontario). These 220 patients were then invited by e-mail to participate in our study, of which 175 (80\%) completed the online study questionnaire. The median time between diagnosis and questionnaire completion was 10 months (5th-95th percentile: 5-16 months). Compared to all eligible cases who did not participate in the study, participating cases were more likely to be female ( $58 \%$ vs. $43 \%$ ) but had similar distributions of age at diagnosis (mean: 43 vs. 42 years), stage (III or IV: $60 \%$ vs. $64 \%$ ), and tumor subsite (distal colon or rectum: $76 \%$ vs. $74 \%$ ).

\section{Control recruitment}

Population-based controls, defined as Ontario residents aged 20-49 years (frequency-matched to case estimates by sex and 5-year age group) with no history of CRC, were recruited by the Institute for Social Research at York University (Toronto, Ontario) in 2019 using modified random digit dialing methods. A sampling frame of telephone numbers was constructed using provincial directories and commercially available lists, as well as numbers on either side of listed numbers. Approximately 53,000 randomly selected households were telephoned to identify eligible controls. Of the 1,800 households for which an eligible person was identified, 640 expressed interest and provided their contact information. We then invited these 640 persons by e-mail to participate in our study, of which 253 (40\%) completed the online questionnaire. 


\section{Data collection}

An invitation letter that included a URL link to access the web-based study questionnaire was e-mailed to all eligible cases and controls who provided initial opt-in consent (cases) or expressed interest in study participation (controls). Generally, non-respondents were followed up by e-mail after 3 weeks, then by telephone 2 weeks later, and a final followup e-mail at week 7.

The online questionnaire collected self-reported information on sociodemographics (age, sex, race/ethnicity, birth country, education, income, occupation, rural/urban residence), family history of CRC, personal medical history (type 2 diabetes, IBD, other chronic inflammatory conditions, allergy/asthma), prior medical procedures (sigmoidoscopy/colonoscopy, CT scans), medication use (aspirin/nonsteroidal anti-inflammatory drugs [NSAID], laxatives, oral antibiotics), female reproductive history (parity, age at first pregnancy, oral contraceptive [OC] use, menopausal status), body mass index (BMI; weight [kg] divided by height [m] squared), smoking status, secondhand smoke exposure, alcohol consumption, physical activity (adapted from the previously validated Godin-Shephard Leisure-Time Physical Activity Questionnaire, which asked participants to report the number of times they engaged in strenuous [heart beats rapidly, heavy breathing, sweating; e.g., running, jogging, hockey, soccer, squash, basketball, football, cross-country skiing, skating, vigorous swimming, aerobics, vigorous bicycling, spinning, judo] or moderate [slight increase in heart rate and breathing, light sweating; e.g., fast walking, baseball, tennis, easy bicycling, volleyball, badminton, easy swimming, dancing, hiking, downhill skiing, weightlifting] exercise/physical activity for $>15$ min during a typical 7-day week [27, 28]), sedentary time (number of hours spent sitting on a typical day at work, school, home, in a car/bus/ train, and during leisure time [e.g., watching TV, playing video games, using computer, reading, socializing], averaged over weekdays and weekend days), supplement use (calcium, antacid, vitamin D/cod liver oil, prebiotics, probiotics, folic acid), and consumption of various foods (fruits, vegetables, high-fiber/wholegrain foods, red meat, processed meat, sugary desserts [e.g., candy, chocolate bars, cake, cookies, ice cream], fast food [e.g., burger, fries, taco, pizza, instant ramen noodles], canned food, processed snacks [e.g., chips, crackers, white bread, sugary cereals]), beverages (sugary drinks [e.g., non-diet soft drinks, vitamin drinks, energy drinks, specialty coffee with syrup such as mocha], coffee/tea, water), and sugar substitutes (artificial sweeteners, agave syrup). Dietary intake was assessed as frequency of consumption (daily or weekly) for specified serving sizes as applicable, with detailed examples provided in the questionnaire to aid participant response (e.g., one serving of fruit is: 1 medium-sized fresh fruit, 1/2 cup of chopped, cooked, or canned fruit, $1 / 4$ cup of dried fruit, or $1 / 2$ cup of fruit juice). To ensure pre-diagnosis information was collected, participants were asked to report dietary habits and other lifestyle factors (e.g., alcohol consumption, physical activity, sedentary time) for the time period " 2 years ago". For most other variables, including prior medical diagnoses and procedures and medication or supplement use, participants were asked to only report those occurring at least 2 years before questionnaire completion. Variables are clearly defined in table footnotes.

\section{Western-like dietary pattern derivation}

In addition to examining specific foods/beverages (listed above) as potential EO-CRC risk factors, a composite dietary pattern score was derived as a measure of overall diet quality. This "Western-like" dietary pattern score was derived based on the consumption of six non-beneficial (red meat, processed meat, sugary drinks, sugary desserts, fast food, and processed snacks) and three beneficial (fruits, vegetables, and high-fiber/wholegrain foods) components commonly identified in previous studies [29]. For each non-beneficial component, participants in the first (lowest), second, third, and fourth (highest) quartile of intake (roughly categorized based on distribution among controls) were assigned a value of $0,1,2$, and 3, respectively. Conversely, for each beneficial component, the quartiles were reverse coded (i.e., $3,2,1$, and 0 for the first, second, third, and fourth quartile, respectively). The final score was calculated by summing up values across all nine components, with higher scores (range: 0-27) indicating a more Westernized dietary pattern.

\section{Statistical analysis}

Descriptive statistics, including frequencies and proportions, were computed by case-control status for all variables. In general, variables were categorized based on the original categories in the study questionnaire, standard cut-off points (e.g., BMI, physical activity), meaningful cut-offs used in previous studies for ease of interpretation and comparison (e.g., food and beverage consumption), and/or statistical considerations given the distribution among study subjects.

Binary logistic regression analyses were performed to estimate associations between each variable of interest and EO-CRC risk, reported as age- and sex-adjusted and multivariable-adjusted odds ratios (OR), with $95 \%$ confidence intervals (CI). Multivariable models were constructed with the main variable of interest and a list of a priori covariates that included age, sex, family history of CRC, aspirin/ NSAID use, smoking, physical activity, BMI, alcohol consumption, red/processed meat intake, fruit and vegetable intake, high-fiber food intake, and calcium supplement use. These covariates were chosen as they were established risk/ 
protective factors for overall CRC $[14,30]$, and they were retained in all models regardless of statistical significance and influence on the effect estimates of other variables. Notably, while diabetes and IBD are also known CRC risk factors, they were not forced into models due to the small proportion of participants reporting each of these conditions $(<5 \%)$. Instead, diabetes and IBD, along with several variables not typically considered as CRC risk factors (race/ ethnicity, education, income), were evaluated as potential confounders and included in the final model only if their removal resulted in $\geq 15 \%$ change in the OR estimate of the main variable.

Analyses were further stratified by sex, and statistical significance of the interaction between sex and each variable was evaluated using the likelihood ratio test. As an exploratory analysis to investigate potential etiologic heterogeneity by anatomical subsite, polytomous logistic regression was used to estimate associations between each variable and CRC tumor subsite-specific risks (proximal colon and distal colon/rectum).

Analyses were performed using SAS, version 9.4 (SAS Institute Inc., Cary, NC). Statistical significance was evaluated at $p<0.05$, and all tests were two-sided.

\section{Results}

Table 1 presents sociodemographic and clinical characteristics of the $175 \mathrm{EO}-\mathrm{CRC}$ cases and 253 controls. The mean ages of cases and controls were 43 and 40 years, respectively. The majority of cases and controls were female (57\%), white $(81 \%)$, had at least a college or university degree (82\%), and resided in an urban area for most of their lives (81\%). Of cases with available data, 24\%, 28\%, and $48 \%$ had cancer of the proximal colon, distal colon, and rectum, respectively, $11 \%$ had MSI-high tumors, and $60 \%$ were diagnosed at stage III or IV.

Table 2 presents associations between family and personal medical history and EO-CRC risk. Family history of $\mathrm{CRC}$ in a first- or second-degree relative was associated with an increased risk of EO-CRC (multivariable-adjusted OR [MVOR] 2.37; 95\% CI 1.47-3.84), with a stronger association if at least one relative was diagnosed before 50 years of age (MVOR 3.35; 95\% CI 1.25-8.98). Personal history of type 2 diabetes showed a positive but non-significant association with EO-CRC risk (MVOR 1.75; 95\% CI 0.57-5.32), while history of allergy/asthma was associated with a statistically significant reduced risk (MVOR 0.62; 95\% CI 0.39-0.98), especially when diagnosed before age 10 (MVOR 0.43; 95\% CI 0.21-0.88). Furthermore, compared to those who had never had a CT scan, those with 1 or 2 scans had a significantly lower risk (MVOR 0.30; 95\% CI 0.16-0.57), whereas those with $\geq 3$ CT scans had a suggestive increased risk (MVOR 2.15; 95\% CI 0.96-4.81). No associations were observed for ever (vs. never) use of aspirin/NSAID, laxatives, or oral antibiotics; however, oral antibiotic use during childhood alone was associated with a statistically significant lower risk (MVOR 0.29; 95\% CI 0.09-0.90). Among females, parity was inversely associated with EO-CRC risk ( $\geq 3$ vs. nulliparity, MVOR 0.29; 95\% CI 0.11-0.76; $p_{\text {trend }}=0.01$ ), whereas positive (albeit non-significant) associations were observed for older age at first pregnancy ( $\geq 30$ vs. $<30$ years, MVOR 1.90 ) and being postmenopausal (MVOR 2.19).

Table 3 presents associations between lifestyle factors and EO-CRC risk. While no association was observed for ever (vs. never) smoking, those in the first tertile of pack-years had a significantly elevated risk compared to never smokers (MVOR 1.94; 95\% CI 1.04-3.60). Longer sedentary time was associated with a statistically significant increased risk of EO-CRC ( $\geq 10$ vs. $<5$ h/day, MVOR 1.93 ; 95\% CI $\left.1.02-3.65 ; p_{\text {trend }}=0.049\right)$, whereas BMI at early age $20 \mathrm{~s}$ and 2 years ago both showed a suggestive inverse association (both $\left.p_{\text {trend }}=0.06\right)$, with respective MVORs of $0.43(95 \%$ CI $0.20-0.90)$ and 0.59 (95\% CI 0.34-1.01) for obesity. Furthermore, despite the lack of statistical significance, longer duration of secondhand smoke exposure and being less physically active tended toward higher risk, while alcohol consumption showed no associations.

Table 4 presents associations between dietary factors and EO-CRC risk. Greater consumption of sugary drinks ( $\geq 7$ vs. $<1$ drinks/week, MVOR 2.99; 95\% CI 1.57-5.68; $p_{\text {trend }}=0.002$ ), sugary desserts (3-6 vs. $<3$ times/week [middle category only], MVOR 2.28; 95\% CI 1.28-4.04), and a higher Western-like dietary pattern score (quartile 4 vs. 1 , MVOR 1.92; 95\% CI 1.01-3.66; $p_{\text {trend }}=0.047$ ) were associated with elevated risks of EO-CRC. Statistically significant associations were not observed for fruits, vegetables, highfiber foods, red meat, or processed meat, although greater vegetable consumption showed a tendency toward lower risk ( $\left.p_{\text {trend }}=0.08\right)$. In addition, more frequent consumption of fast food ( $\geq 2$ vs. $<1$ times/week, MVOR 1.84; 95\% CI $\left.0.98-3.46 ; p_{\text {trend }}=0.07\right)$ and canned food $(\geq 3$ vs. $<1$ times/ week, MVOR 1.70; 95\% CI 0.95-3.05; $p_{\text {trend }}=0.09$ ) showed suggestive associations with increased EO-CRC risk. Of all supplements assessed, calcium was the only one associated with a statistically significant lower risk (ever vs. never use, MVOR 0.53; 95\% CI 0.31-0.92).

Sex-stratified analyses revealed similar associations among males and females for most variables, although statistical significance was generally not achieved (Supplementary Tables S1-S3). Notably, greater sugary drink consumption was associated with statistically significantly increased risk of EO-CRC in both males and females ( $p_{\text {trend }}<0.05$ ). Moreover, despite the lack of a significant interaction $\left(p_{\text {interaction }}=0.08\right)$, coffee/tea consumption was positively 
Table 1 Sociodemographic and clinical characteristics of earlyonset colorectal cancer cases and controls aged 20-49 years, Ontario, Canada, 2018-2019

\begin{tabular}{|c|c|c|c|}
\hline \multirow[t]{2}{*}{ Characteristics } & \multirow{2}{*}{$\begin{array}{l}\begin{array}{l}\text { Cases } \\
(n=175)\end{array} \\
n^{\mathrm{a}} \\
(\%)\end{array}$} & \multirow{2}{*}{$\begin{array}{l}\text { Controls } \\
(n=253)\end{array}$} & \multirow[t]{2}{*}{$\begin{array}{l}\text { Age- and sex-adjusted } \mathrm{OR}^{\mathrm{b}} \\
(95 \% \mathrm{CI})\end{array}$} \\
\hline & & & \\
\hline \multicolumn{4}{|l|}{ Age, years } \\
\hline Mean (SD) & $\begin{array}{l}43.1 \\
(5.6)\end{array}$ & $\begin{array}{l}40.1 \\
(7.9)\end{array}$ & N/A \\
\hline \multicolumn{4}{|l|}{ Age group, years } \\
\hline $20-29$ & $\begin{array}{l}6 \\
(3)\end{array}$ & $\begin{array}{l}36 \\
(14)\end{array}$ & N/A \\
\hline $30-34$ & $\begin{array}{l}11 \\
(6)\end{array}$ & $\begin{array}{l}18 \\
(7)\end{array}$ & \\
\hline $35-39$ & $\begin{array}{l}23 \\
(13)\end{array}$ & $\begin{array}{l}35 \\
(14)\end{array}$ & \\
\hline $40-44$ & $\begin{array}{l}38 \\
(22)\end{array}$ & $\begin{array}{l}69 \\
(27)\end{array}$ & \\
\hline $45-49$ & $\begin{array}{l}97 \\
(55)\end{array}$ & $\begin{array}{l}95 \\
(38)\end{array}$ & \\
\hline \multicolumn{4}{|l|}{ Sex } \\
\hline Male & $\begin{array}{l}74 \\
(42)\end{array}$ & $\begin{array}{l}112 \\
(44)\end{array}$ & N/A \\
\hline Female & $\begin{array}{l}101 \\
(58)\end{array}$ & $\begin{array}{l}141 \\
(56)\end{array}$ & \\
\hline \multicolumn{4}{|l|}{ Race/ethnicity } \\
\hline White & $\begin{array}{l}141 \\
(81)\end{array}$ & $\begin{array}{l}202 \\
(80)\end{array}$ & 1.00 \\
\hline East/Southeast Asian & $\begin{array}{l}16 \\
(9)\end{array}$ & $\begin{array}{l}16 \\
(6)\end{array}$ & $1.50(0.71-3.15)$ \\
\hline South Asian & $\begin{array}{l}5 \\
(3)\end{array}$ & $\begin{array}{l}16 \\
(6)\end{array}$ & $0.57(0.20-1.66)$ \\
\hline Other $^{\mathrm{c}}$ & $\begin{array}{l}13 \\
(7)\end{array}$ & $\begin{array}{l}17 \\
(7)\end{array}$ & $1.03(0.48-2.21)$ \\
\hline \multicolumn{4}{|l|}{ Country of birth } \\
\hline Canada & $\begin{array}{l}146 \\
(83)\end{array}$ & $\begin{array}{l}184 \\
(73)\end{array}$ & 1.00 \\
\hline Outside Canada & $\begin{array}{l}29 \\
(17)\end{array}$ & $\begin{array}{l}69 \\
(27)\end{array}$ & $0.51(0.31-0.84)$ \\
\hline \multicolumn{4}{|l|}{ Highest level of education } \\
\hline High school graduate or less & $\begin{array}{l}32 \\
(18)\end{array}$ & $\begin{array}{l}46 \\
(19)\end{array}$ & 1.00 \\
\hline College or university degree & $\begin{array}{l}98 \\
(56)\end{array}$ & $\begin{array}{l}138 \\
(56)\end{array}$ & $0.97(0.57-1.66)$ \\
\hline Post-graduate degree & $\begin{array}{l}45 \\
(26)\end{array}$ & $\begin{array}{l}64 \\
(26)\end{array}$ & $0.98(0.54-1.80)$ \\
\hline \multicolumn{4}{|l|}{ Annual household income ${ }^{\mathrm{d}}, \mathrm{CAD} \$$} \\
\hline$<\$ 30,000$ & $\begin{array}{l}9 \\
(5)\end{array}$ & $\begin{array}{l}23 \\
(10)\end{array}$ & 1.00 \\
\hline$\$ 30,000-\$ 69,999$ & $\begin{array}{l}29 \\
(18)\end{array}$ & $\begin{array}{l}44 \\
(20)\end{array}$ & $1.56(0.62-3.93)$ \\
\hline$\$ 70,000-\$ 100,000$ & $\begin{array}{l}38 \\
(23)\end{array}$ & $\begin{array}{l}46 \\
(21)\end{array}$ & $1.91(0.77-4.70)$ \\
\hline$>\$ 100,000$ & $\begin{array}{l}88 \\
(54)\end{array}$ & $\begin{array}{l}108 \\
(49)\end{array}$ & $1.85(0.80-4.29)$ \\
\hline Unknown (prefer not to answer) & 11 & 32 & \\
\hline \multicolumn{4}{|l|}{ Occupation $^{\mathrm{d}}$} \\
\hline Professional $^{\mathrm{e}}$ & $\begin{array}{l}63 \\
(36)\end{array}$ & $\begin{array}{l}93 \\
(37)\end{array}$ & 1.00 \\
\hline Managerial and Administrative ${ }^{f}$ & $\begin{array}{l}33 \\
(19)\end{array}$ & $\begin{array}{l}41 \\
(16)\end{array}$ & $1.12(0.63-1.98)$ \\
\hline
\end{tabular}


Table 1 (continued)

\begin{tabular}{|c|c|c|c|}
\hline \multirow[t]{2}{*}{ Characteristics } & \multirow{2}{*}{$\begin{array}{l}\begin{array}{l}\text { Cases } \\
(n=175)\end{array} \\
n^{\mathrm{a}} \\
(\%)\end{array}$} & \multirow{2}{*}{$\begin{array}{l}\text { Controls } \\
(n=253)\end{array}$} & \multirow[t]{2}{*}{$\begin{array}{l}\text { Age- and sex-adjusted OR } \\
(95 \% \mathrm{CI})\end{array}$} \\
\hline & & & \\
\hline Sales and Services ${ }^{\mathrm{g}}$ & $\begin{array}{l}25 \\
(14)\end{array}$ & $\begin{array}{l}34 \\
(13)\end{array}$ & $1.11(0.60-2.06)$ \\
\hline Clerical $^{\mathrm{h}}$ & $\begin{array}{l}22 \\
(13)\end{array}$ & $\begin{array}{l}21 \\
(8)\end{array}$ & $1.46(0.73-2.94)$ \\
\hline Manufacturing, Agriculture, and Trades $\mathrm{s}^{\mathrm{i}}$ & $\begin{array}{l}17 \\
(10)\end{array}$ & $\begin{array}{l}26 \\
(10)\end{array}$ & $1.08(0.53-2.21)$ \\
\hline Student/not employed & $\begin{array}{l}7 \\
(4)\end{array}$ & $\begin{array}{l}27 \\
(11)\end{array}$ & $0.53(0.21-1.37)$ \\
\hline Other/unspecified & $\begin{array}{l}8 \\
(5)\end{array}$ & $\begin{array}{l}11 \\
(4)\end{array}$ & $1.01(0.38-2.67)$ \\
\hline \multicolumn{4}{|l|}{ Usual residence during most of life } \\
\hline Urban & $\begin{array}{l}140 \\
(81)\end{array}$ & $\begin{array}{l}202 \\
(81)\end{array}$ & 1.00 \\
\hline Rural & $\begin{array}{l}32 \\
(19)\end{array}$ & $\begin{array}{l}47 \\
(19)\end{array}$ & $0.98(0.59-1.63)$ \\
\hline \multicolumn{4}{|l|}{ Anatomical subsite ${ }^{j}$} \\
\hline Proximal colon & $\begin{array}{l}41 \\
(24)\end{array}$ & N/A & N/A \\
\hline Distal colon & $\begin{array}{l}49 \\
(28)\end{array}$ & & \\
\hline Rectum & $\begin{array}{l}84 \\
(48)\end{array}$ & & \\
\hline \multicolumn{4}{|l|}{ MSI status ${ }^{\mathrm{k}}$} \\
\hline Microsatellite stable or MSI-low & $\begin{array}{l}122 \\
(89)\end{array}$ & N/A & N/A \\
\hline MSI-high & $\begin{array}{l}15 \\
(11)\end{array}$ & & \\
\hline Unknown & 38 & & \\
\hline \multicolumn{4}{|l|}{ Stage at diagnosis ${ }^{1}$} \\
\hline Stage I & $\begin{array}{l}28 \\
(19)\end{array}$ & N/A & N/A \\
\hline Stage II & $\begin{array}{l}30 \\
(21)\end{array}$ & & \\
\hline Stage III & $\begin{array}{l}57 \\
(39)\end{array}$ & & \\
\hline Stage IV & $\begin{array}{l}31 \\
(21)\end{array}$ & & \\
\hline Unknown & 29 & & \\
\hline
\end{tabular}

$C A D$ Canadian Dollar; $C I$ confidence interval; $M S I$ microsatellite instability; N/A not applicable; $O R$ odds ratio; $S D$ standard deviation

aNumbers may not sum up to totals due to missing data. An "unknown" category is shown for variables with $>5 \%$ missing data

${ }^{\mathrm{b}}$ Adjusted for age (continuous, years; age at diagnosis for cases and at questionnaire completion for controls) and sex

${ }^{\mathrm{c}}$ Includes Black $(n=9)$, Aboriginal (First Nations, Métis, or Inuit; $\left.n=6\right)$, and other race/ethnicity $(n=15)$. Numbers broken down by case/control status are not shown due to small cell sizes

${ }^{\mathrm{d}}$ Two years ago

${ }^{\text {e}}$ Professional occupations in natural and applied sciences, health, education, law and social, community and government services

${ }^{\mathrm{f}}$ Management, business, finance and administration occupations

${ }^{\mathrm{g}}$ Sales and service occupations, including occupations related to the hospitality and tourism industries

${ }^{\mathrm{h}}$ Administrative and office support occupations

${ }^{i}$ Occupations in manufacturing (e.g., metal, glass, chemicals, wood, pulp, textile), agriculture and natural resources (e.g., farming, fishing, forestry), construction, trades, transport and equipment operation 
Table 1 (continued)

\begin{abstract}
${ }^{\mathrm{j}}$ Based on International Classification of Diseases for Oncology, 3rd Edition (ICD-O-3) topography codes (proximal colon: cecum [C18.0], ascending colon [C18.2], hepatic flexure of colon [C18.3], transverse colon [C18.4], and splenic flexure of colon [C18.5]; distal colon: descending colon [C18.6] and sigmoid colon [C18.7]; rectum: rectosigmoid junction [C19.9] and rectum, not otherwise specified [C20.9])

${ }^{k}$ Based on immunohistochemistry staining results for DNA mismatch repair proteins (MLH1, MSH2, MSH6, and PMS2). Cases were considered microsatellite stable or MSI-low if they had normal (intact) nuclear staining for all four proteins, else they were considered MSI-high if they had abnormal (deficient) staining for any of the four proteins

${ }^{\mathrm{l}}$ Determined based on multiple sources of staging information, including pathological staging and clinical staging (pathological stage was given priority where available), in accordance with the American Joint Committee on Cancer (AJCC) tumor-node-metastases (TNM) staging system, 8th Edition
\end{abstract}

associated with EO-CRC risk among males $(\geq 3$ vs. $<1$ cups/day, MVOR 3.08; 95\% CI 1.14-8.33; $\left.p_{\text {trend }}=0.02\right)$ but not females (MVOR 0.99; 95\% CI 0.40-2.43; $p_{\text {trend }}=0.97$ ). Another notable sex difference is the tendency toward increased risk for greater red meat consumption among males ( $\geq 5$ vs. $<2$ servings/week, MVOR 2.64; 95\% CI $\left.0.84-8.33 ; p_{\text {trend }}=0.09\right)$, as compared to the non-significant inverse association among females (MVOR 0.68; 95\% CI $\left.0.31-1.47 ; p_{\text {trend }}=0.56\right)\left(p_{\text {interaction }}=0.09\right)$.

When analyses were performed by CRC subsite (Supplementary Tables S4-S6), similar patterns of associations were generally observed for proximal colon and distal colon/rectal cancer, with a few exceptions. Notably, calcium supplement use was inversely associated with distal colon/rectal (MVOR 0.40; 95\% CI 0.22-0.76), but not proximal colon (MVOR 1.01; 95\% CI 0.43-2.37), cancer $\left(p_{\text {heterogeneity }}=0.06\right)$, and antacid use was differentially associated with risk of proximal colon (MVOR 2.20; 95\% CI 1.04-4.63) and distal colon/rectal (MVOR 0.64; 95\% CI $0.39-1.06)$ cancer $\left(p_{\text {heterogeneity }}=0.003\right)$.

\section{Discussion}

This population-based case-control study found that family history of CRC, longer sedentary time, greater consumption of sugary drinks, and a more Westernized dietary pattern were each associated with a statistically significant increased risk of EO-CRC, whereas history of allergy/asthma, oral antibiotic use during childhood alone, being overweight, calcium supplement use, and greater parity in females were each associated with a statistically significant reduced risk. In addition, more frequent consumption of fast food and canned food showed suggestive associations with increased EO-CRC risk, while greater vegetable consumption tended toward lower risk. Having $\geq 3$ CT scans (vs. never) was also associated with a suggestive increased risk, although there was a lack of consistent trend across categories.

While our study confirmed previous findings that family history of CRC is a strong risk factor for EO-CRC $[15,16$, 19-23], we did not observe an association for several factors known to affect overall CRC risk, including smoking, alcohol, and aspirin/NSAID. Findings from previous EOCRC studies regarding these factors have also been inconsistent and inconclusive [15, 16, 19-21, 23, 24, 31], although a recent meta-analysis of EO-CRC risk factors reported a significant association for alcohol consumption (heavy vs. non-drinkers) based on only three studies (pooled relative risk [RR] 1.71; 95\% CI 1.62-1.80) [15]. A possible explanation for the general lack of associations may be the long latency required $[32,33]$ such that established associations with overall (primarily older-onset) CRC may not hold up for EO-CRC. For example, it has been suggested that cigarette smoking plays a stronger role in the initiation of colorectal adenoma, and that its association with increased CRC risk becomes apparent only after a sufficiently long lag period [33]. This hypothesis is supported by the relatively consistent association observed between smoking (as well as alcohol intake) and early-onset colorectal adenoma [34-39]. Meanwhile, an association between smoking (or tobacco use) and EO-CRC risk was only seen in three cohort studies relying on EHR data [19-21], but not in three other case-control studies [23, 24, 31] or the meta-analysis (pooled RR 1.35; 95\% CI 0.81-2.25) [15]. Reasons for the increased risk we observed for tertile 1 (vs. never) of smoking pack-years, but not higher tertiles, are unclear and may be a spurious finding. Similarly, only two $[16,20]$ of five $[16,19-21,31]$ previous studies revealed a possible link between alcohol and EO-CRC risk, including a case-control study assessing self-reported consumption [16] and an EHR-based cohort study assessing alcohol-related diagnoses [20]. Furthermore, contrary to our null finding, the only previous study to assess aspirin use in relation to EO-CRC risk (case-control study of US veterans) reported an OR of 0.66 (95\% CI 0.52-0.84) [24]. Given the chemoprotective potential of aspirin/NSAID against CRC [40-42], further evaluation of their association with EO-CRC risk is needed.

Despite the lack of statistical significance, we found that personal history of diabetes was associated with a nearly two-fold increase in odds of EO-CRC. While there is convincing evidence of diabetes (and underlying insulin resistance and hyperinsulinemia) as a risk factor for overall CRC [43-45], evidence for EO-CRC remains inconclusive, with some [21, 22, 46], but not all [16, 19, 23, 24], studies 
Table 2 Associations of family history, medical history, and female reproductive factors with risk of early-onset colorectal cancer, Ontario, Canada, 2018-2019

\begin{tabular}{|c|c|c|c|c|}
\hline \multirow[t]{2}{*}{ Variables } & \multirow{2}{*}{$\begin{array}{l}\text { Cases } \\
(n=175) \\
n^{\mathrm{a}} \\
(\%)\end{array}$} & \multirow{2}{*}{$\begin{array}{l}\text { Controls } \\
(n=253) \\
n^{\mathrm{a}} \\
(\%)\end{array}$} & \multirow[t]{2}{*}{$\begin{array}{l}\text { Age- and sex-adjusted } \mathrm{OR}^{\mathrm{b}} \\
(95 \% \mathrm{CI})\end{array}$} & \multirow[t]{2}{*}{$\begin{array}{l}\text { Multivariable- } \\
\text { adjusted OR } \\
(95 \% \mathrm{CI})\end{array}$} \\
\hline & & & & \\
\hline \multicolumn{5}{|l|}{ Family history of CRC ${ }^{\mathrm{d}}$} \\
\hline No & $\begin{array}{l}91 \\
(58)\end{array}$ & $\begin{array}{l}169 \\
(76)\end{array}$ & 1.00 & 1.00 \\
\hline Yes & $\begin{array}{l}65 \\
(42)\end{array}$ & $\begin{array}{l}52 \\
(24)\end{array}$ & $2.20(1.40-3.45)$ & $2.37(1.47-3.84)$ \\
\hline \multicolumn{5}{|c|}{ Age of youngest relative at diagnosis } \\
\hline$<50$ years & $\begin{array}{l}12 \\
(8)\end{array}$ & $\begin{array}{l}9 \\
(4)\end{array}$ & $2.77(1.09-7.01)$ & $3.35(1.25-8.98)$ \\
\hline$\geq 50$ years & $\begin{array}{l}53 \\
(34)\end{array}$ & $\begin{array}{l}43 \\
(19)\end{array}$ & $2.09(1.29-3.39)$ & $2.22(1.33-3.69)$ \\
\hline Unknown & 19 & 32 & & \\
\hline \multicolumn{5}{|c|}{ Personal medical history ${ }^{\mathrm{e}}$} \\
\hline \multicolumn{5}{|l|}{ Type 2 diabetes } \\
\hline No & $\begin{array}{l}167 \\
(95)\end{array}$ & $\begin{array}{l}245 \\
(97)\end{array}$ & 1.00 & 1.00 \\
\hline Yes & $\begin{array}{l}8 \\
(5)\end{array}$ & $\begin{array}{l}8 \\
(3)\end{array}$ & $1.32(0.48-3.61)$ & $1.75(0.57-5.32)$ \\
\hline \multicolumn{5}{|c|}{ Chronic inflammatory condition ${ }^{\mathrm{f}}$} \\
\hline No & $\begin{array}{l}162 \\
(93)\end{array}$ & $\begin{array}{l}230 \\
(91)\end{array}$ & 1.00 & 1.00 \\
\hline Yes & $\begin{array}{l}13 \\
\text { (7) }\end{array}$ & $\begin{array}{l}22 \\
(9)\end{array}$ & $0.82(0.40-1.71)$ & $0.89(0.41-1.92)$ \\
\hline \multicolumn{5}{|l|}{ Allergy or asthma } \\
\hline No & $\begin{array}{l}122 \\
(71)\end{array}$ & $\begin{array}{l}151 \\
(60)\end{array}$ & 1.00 & 1.00 \\
\hline Yes & $\begin{array}{l}51 \\
(29)\end{array}$ & $\begin{array}{l}99 \\
(40)\end{array}$ & $0.68(0.44-1.03)$ & $0.62(0.39-0.98)$ \\
\hline \multicolumn{5}{|l|}{ Age at diagnosis } \\
\hline$<10$ years & $\begin{array}{l}13 \\
(8)\end{array}$ & $\begin{array}{l}43 \\
(17)\end{array}$ & $0.43(0.22-0.85)$ & $0.43(0.21-0.88)$ \\
\hline $10-19$ years & $\begin{array}{l}23 \\
(13)\end{array}$ & $\begin{array}{l}34 \\
(14)\end{array}$ & $0.93(0.51-1.69)$ & $0.88(0.46-1.69)$ \\
\hline$\geq 20$ years & $\begin{array}{l}15 \\
(9)\end{array}$ & $\begin{array}{l}22 \\
(9)\end{array}$ & $0.73(0.36-1.49)$ & $0.60(0.28-1.28)$ \\
\hline \multicolumn{5}{|c|}{ Sigmoidoscopy or colonoscopy } \\
\hline No & $\begin{array}{l}149 \\
(85)\end{array}$ & $\begin{array}{l}212 \\
(84)\end{array}$ & 1.00 & 1.00 \\
\hline Yes & $\begin{array}{l}26 \\
(15)\end{array}$ & $\begin{array}{l}40 \\
(16)\end{array}$ & $0.78(0.45-1.35)$ & $0.69(0.38-1.25)$ \\
\hline \multicolumn{5}{|c|}{ Total number of CT scans } \\
\hline Never had a CT scan & $\begin{array}{l}133 \\
(79)\end{array}$ & $\begin{array}{l}165 \\
(70)\end{array}$ & 1.00 & 1.00 \\
\hline $1-2$ & $\begin{array}{l}15 \\
(9)\end{array}$ & $\begin{array}{l}58 \\
(24)\end{array}$ & $0.31(0.16-0.57)$ & $0.30(0.16-0.57)$ \\
\hline$\geq 3$ & $\begin{array}{l}20 \\
(12)\end{array}$ & $\begin{array}{l}14 \\
(6)\end{array}$ & $1.99(0.94-4.23)$ & $2.15(0.96-4.81)$ \\
\hline Unknown & 7 & 16 & & \\
\hline \multicolumn{5}{|c|}{ Middle and/or lower body CT scan } \\
\hline No & $\begin{array}{l}140 \\
(83)\end{array}$ & $\begin{array}{l}194 \\
(83)\end{array}$ & 1.00 & 1.00 \\
\hline
\end{tabular}


Table 2 (continued)

\begin{tabular}{|c|c|c|c|c|}
\hline \multirow[t]{2}{*}{ Variables } & \multirow{2}{*}{$\begin{array}{l}\text { Cases } \\
(n=175) \\
n^{\mathrm{a}} \\
(\%)\end{array}$} & \multirow{2}{*}{$\begin{array}{l}\text { Controls } \\
(n=253)\end{array}$} & \multirow[t]{2}{*}{$\begin{array}{l}\text { Age- and sex-adjusted } \mathrm{OR}^{\mathrm{b}} \\
(95 \% \mathrm{CI})\end{array}$} & \multirow{2}{*}{$\begin{array}{l}\text { Multivariable- } \\
\text { adjusted OR }{ }^{\mathrm{c}} \\
(95 \% \mathrm{CI})\end{array}$} \\
\hline & & & & \\
\hline Yes & $\begin{array}{l}28 \\
(17)\end{array}$ & $\begin{array}{l}39 \\
(17)\end{array}$ & $1.03(0.59-1.78)$ & $1.02(0.57-1.82)$ \\
\hline Unknown & 7 & 20 & & \\
\hline \multicolumn{5}{|l|}{ Medication use } \\
\hline \multicolumn{5}{|l|}{ Regular aspirin or NSAID use ${ }^{g}$} \\
\hline Never & $\begin{array}{l}119 \\
(68)\end{array}$ & $\begin{array}{l}180 \\
(71)\end{array}$ & 1.00 & 1.00 \\
\hline Ever & $\begin{array}{l}56 \\
(32)\end{array}$ & $\begin{array}{l}73 \\
(29)\end{array}$ & $1.06(0.68-1.63)$ & $1.20(0.75-1.92)$ \\
\hline \multicolumn{5}{|l|}{ Regular laxative use $\mathrm{g}^{\mathrm{g}}$} \\
\hline Never & $\begin{array}{l}168 \\
(96)\end{array}$ & $\begin{array}{l}241 \\
(95)\end{array}$ & 1.00 & 1.00 \\
\hline Ever & $\begin{array}{l}7 \\
(4)\end{array}$ & $\begin{array}{l}12 \\
(5)\end{array}$ & $0.79(0.29-2.12)$ & $0.92(0.32-2.59)$ \\
\hline \multicolumn{5}{|l|}{ Oral antibiotic use ${ }^{\mathrm{h}}$} \\
\hline No & $\begin{array}{l}139 \\
(79)\end{array}$ & $\begin{array}{l}191 \\
(75)\end{array}$ & 1.00 & 1.00 \\
\hline Yes & $\begin{array}{l}36 \\
(21)\end{array}$ & $\begin{array}{l}62 \\
(25)\end{array}$ & $0.79(0.49-1.27)$ & $0.78(0.47-1.30)$ \\
\hline \multicolumn{5}{|l|}{ Period of use } \\
\hline Childhood only (child or teenager) & $-^{\mathrm{i}}$ & $\begin{array}{l}22 \\
(9)\end{array}$ & $0.30(0.10-0.91)$ & $0.29(0.09-0.90)$ \\
\hline Adulthood only (age 20 s or later) & & $\begin{array}{l}27 \\
(11)\end{array}$ & $0.81(0.42-1.57)$ & $0.86(0.43-1.70)$ \\
\hline Both childhood and adulthood & $\begin{array}{l}15 \\
(9)\end{array}$ & $\begin{array}{l}13 \\
(5)\end{array}$ & $1.39(0.63-3.05)$ & $1.42(0.60-3.37)$ \\
\hline Reproductive history (females only) & $(n=101)$ & $(n=141)$ & & \\
\hline \multicolumn{5}{|l|}{ Parity $^{\mathrm{j}}$} \\
\hline 0 (nulliparous) & $\begin{array}{l}29 \\
(29)\end{array}$ & $\begin{array}{l}40 \\
(28)\end{array}$ & 1.00 & 1.00 \\
\hline $1-2$ & $\begin{array}{l}60 \\
(59)\end{array}$ & $\begin{array}{l}71 \\
(50)\end{array}$ & $0.65(0.32-1.30)$ & $0.73(0.35-1.52)$ \\
\hline$\geq 3$ & $\begin{array}{l}12 \\
(12)\end{array}$ & $\begin{array}{l}30 \\
(21)\end{array}$ & $0.29(0.12-0.72)$ & $0.29(0.11-0.76)$ \\
\hline \multicolumn{5}{|l|}{ Age at first pregnancy (parous women) ${ }^{\mathrm{j}}$} \\
\hline$<30$ years & $\begin{array}{l}39 \\
(54)\end{array}$ & $\begin{array}{l}66 \\
(65)\end{array}$ & 1.00 & 1.00 \\
\hline$\geq 30$ years & $\begin{array}{l}33 \\
(46)\end{array}$ & $\begin{array}{l}35 \\
(35)\end{array}$ & $1.46(0.78-2.73)$ & $1.90(0.95-3.79)$ \\
\hline \multicolumn{5}{|l|}{ Oral contraceptive use $\mathrm{e}^{\mathrm{k}}$} \\
\hline Never & $\begin{array}{l}25 \\
(25)\end{array}$ & $\begin{array}{l}30 \\
(22)\end{array}$ & 1.00 & 1.00 \\
\hline Ever & $\begin{array}{l}74 \\
(75)\end{array}$ & $\begin{array}{l}107 \\
(78)\end{array}$ & $0.74(0.40-1.38)$ & $0.65(0.32-1.33)$ \\
\hline
\end{tabular}


Table 2 (continued)

\begin{tabular}{|c|c|c|c|c|}
\hline \multirow[t]{2}{*}{ Variables } & \multirow{2}{*}{$\begin{array}{l}\text { Cases } \\
(n=175) \\
n^{\mathrm{a}} \\
(\%)\end{array}$} & $\begin{array}{l}\text { Controls } \\
(n=253)\end{array}$ & \multirow[t]{2}{*}{$\begin{array}{l}\text { Age- and sex-adjusted } \mathrm{OR}^{\mathrm{b}} \\
(95 \% \mathrm{CI})\end{array}$} & \multirow{2}{*}{$\begin{array}{l}\text { Multivariable- } \\
\text { adjusted OR }{ }^{\mathrm{c}} \\
(95 \% \mathrm{CI})\end{array}$} \\
\hline & & $\begin{array}{l}n^{\mathrm{a}} \\
(\%)\end{array}$ & & \\
\hline \multicolumn{5}{|l|}{ Menopausal status ${ }^{1}$} \\
\hline Premenopausal & $\begin{array}{l}84 \\
(83)\end{array}$ & $\begin{array}{l}131 \\
(93)\end{array}$ & 1.00 & 1.00 \\
\hline Postmenopausal & $\begin{array}{l}17 \\
(17)\end{array}$ & $\begin{array}{l}10 \\
(7)\end{array}$ & $1.90(0.80-4.53)$ & $2.19(0.85-5.64)$ \\
\hline
\end{tabular}

$C I$ confidence interval; $C R C$ colorectal cancer; $C T$ computed tomography; $N S A I D$ nonsteroidal anti-inflammatory drug; $O R$ odds ratio

${ }^{a}$ Numbers may not sum up to totals due to missing data. An "unknown" category is shown for variables with $>5 \%$ missing data

${ }^{\mathrm{b}}$ Adjusted for age (continuous, years; age at diagnosis for cases and at questionnaire completion for controls) and sex

${ }^{\mathrm{c}}$ Adjusted for age (continuous, years), sex, family history of CRC (no, yes, unknown), regular aspirin/NSAID use (never/ever), smoking (never/ ever), physical activity (active, somewhat active, insufficiently active), BMI (continuous, $\mathrm{kg} / \mathrm{m}^{2}$ ), alcohol consumption (<once/month, $1-3$ times/month, 1-6 times/week, daily), red/processed meat intake (continuous, servings/week), total fruit and vegetable intake (continuous, servings/day), high-fiber food intake (continuous, servings/day), and calcium supplement use (never/ever)

${ }^{\mathrm{d}}$ Among any first- or second-degree blood relative

${ }^{\mathrm{e}}$ Based only on diagnoses or medical procedures occurring at least 2 years before questionnaire completion

${ }^{\mathrm{f}}$ Includes inflammatory bowel disease (Crohn's disease and ulcerative colitis), celiac disease, rheumatoid arthritis, psoriasis, lupus, and other chronic inflammatory condition (not including allergy or asthma)

${ }^{\mathrm{g}}$ Ever taken the medication regularly (at least twice per week for one month or longer) before 2 years ago

${ }^{\text {h}}$ Ever used oral antibiotics repeatedly ( $\geq 2$ courses per year) or for an extended period of time ( $>1$ month) before 2 years ago

${ }^{\mathrm{i}}$ Not reported due to small cell counts $(n<5$ for at least one of the cells)

${ }^{\mathrm{j}}$ Based on pregnancies lasting for 6 months or longer. Age at pregnancy was assessed among parous women only (72 cases and 101 controls)

${ }^{\mathrm{k}}$ Ever used oral hormonal contraceptives for at least one year before 2 years ago

${ }^{l}$ Females were classified as premenopausal if they had menstrual periods in the last 2 years and postmenopausal if they had stopped menstruating for at least one year before CRC diagnosis (cases) or questionnaire completion (controls) due to natural menopause or surgery to remove the uterus and/or ovaries

reporting a statistically significant association between diabetes and increased EO-CRC risk. Notably, in a Swedish nationwide cohort study that included over 100,000 diabetic individuals diagnosed before age 50, diabetes was associated with a 1.9 -fold $(95 \%$ CI 1.6-2.3) increase in risk of EO-CRC [22]. The small number of diabetic subjects in our and several other studies may have reduced statistical power to detect an association. Additional larger studies are warranted to better evaluate the role of diabetes in EO-CRC etiology, especially given increasing prevalence of diabetes diagnosed in younger persons and potential implications for CRC screening guidelines [22, 47].

Our finding of a positive association between average daily sitting time and EO-CRC risk suggests that sedentary behavior may be a contributor to CRC development among young adults. This corroborates the association observed between prolonged sedentary TV viewing time and increased EO-CRC risk among female nurses in the Nurses' Health Study [18], although our study further provides evidence for total sedentary time, regardless of activity type, in both males and females. The non-significant association between leisure time physical activity and EO-CRC risk in our study is also comparable to the only other study to have evaluated this association [16]. Meanwhile, an older case-control study among young white men in Los Angeles County reported a suggestive increased risk of EO-CRC associated with lower occupational activity level [31]. Given relatively strong evidence of physical inactivity and sedentary behavior as risk factors for overall CRC [14, 30, 48, 49], and the possibility that these behaviors (and their effects) start early in life [50], there is a need to further assess their roles in EO-CRC etiology.

The rising incidence of EO-CRC has sometimes been attributed to the obesity epidemic [51]. A recent meta-analysis of 7 studies identified obesity as a significant risk factor for EO-CRC (pooled RR 1.54; 95\% CI 1.01-2.35); however, cross-sectional studies were included in the analysis and considerable heterogeneity was detected across studies [15]. The suggestive inverse association between BMI and EO-CRC risk in our study contradicts results from the metaanalysis [15], as well as those from the Nurses' Health Study [17] and analyses of a large EHR database in the US [20, 21], which reported increased risks associated with obesity. Similar to our study, a case-control study of US veterans reported an association between overweight/obesity and reduced risk of EO-CRC [24], while two other case-control 
studies [16, 23] and a prospective cohort study of African American women [52] reported no associations. While weight loss may be an early symptom of CRC and a possible explanation for the inverse association observed between BMI and EO-CRC risk [24], it is unlikely since a similar association was also observed for BMI at early age 20s in our study. Mechanisms underlying obesity's role in CRC etiology are complex and likely involve inflammation, insulin resistance, and alterations in adipocytokines, sex hormones, and intestinal microbiota [53, 54]. Future studies examining early-life body fatness (beyond BMI) and related biomarkers may shed light on these conflicting results $[12,55]$.

Our study is one of the few to examine dietary factors associated with EO-CRC, especially foods/beverages more commonly consumed by recent birth cohorts. Previously, a European case-control study reported that higher processed meat (but not red meat) intake was associated with increased EO-CRC risk, whereas higher intakes of fruits and vegetables were associated with reduced risk [16]. Our results for foods typically known to affect CRC risk (i.e., red/processed meat, fruits/vegetables, fiber/wholegrain) were not statistically significant; however, the suggestive inverse association between vegetable consumption and EO-CRC risk requires verification in larger studies. Likewise, the association between red meat consumption and increased EO-CRC risk in males but not females warrants further investigation, for example, in terms of sex differences in specific meats consumed and cooking method and doneness preferences. More importantly, the association observed for a Western dietary pattern score suggests that poor overall diet quality may be a risk factor for EO-CRC. Western diet, characterized by low-fiber and high-fat/sugar consumption, has been shown to induce inflammation and gut dysbiosis $[25,56,57]$ and was associated with increased risk of early-onset colorectal adenoma in a recent cohort study [58]. Our study further suggests associations between processed foods and beverages - hallmarks of a Western diet - and increased EO-CRC risk. In particular, the strong association between sugary drinks and EO-CRC risk supports the hypothesis that highfructose corn syrup (main sweetener in beverages since the 1980s), along with its negative impacts on insulin sensitivity and gut microbiota, may play a role in EO-CRC etiology $[25,59]$. Evidence from animal studies also suggests that high-fructose corn syrup can promote intestinal tumorigenesis and possibly accelerate progression from precursors to CRC [60]. Moreover, although mechanisms remain to be elucidated, the suggestive associations observed for sugary desserts, fast food, and canned foods are likely mediated by the high content of refined sugars, salt, and saturated fats, as well as various chemicals added during the flavoring or processing (e.g., monosodium glutamate, titanium dioxide, synthetic food dyes) and packaging (e.g., bisphenol A) of these foods [25, 61]. In addition, given increasing use of low-calorie sugar substitutes (e.g., sucralose) [62] and their potential role in modifying gut microbiota $[59,63]$, further research is reasonable as our findings for artificial sweeteners were inconclusive. Our finding of a positive association between coffee/tea consumption and EO-CRC risk in males also requires additional investigation, as these beverages are generally suggested as protective against cancer, although evidence of their associations with overall CRC risk remains inconclusive [30, 64, 65].

To our knowledge, this is the first study to assess dietary supplements in relation to EO-CRC risk. The finding of a protective effect of calcium supplement use is consistent with evidence for overall CRC [30, 66, 67] and may have implications for chemoprevention research [40]. Conversely, calcium intake from foods alone was not associated with EO-CRC risk according to a case-control study that examined dietary intakes of micronutrients [16], possibly suggesting that higher doses of calcium obtained from supplements may be more relevant for EO-CRC prevention, although future studies assessing both dietary and supplemental nutrient intakes are needed. Our results further suggest that the protective effect of calcium supplement may be confined to distal colon/rectal cancer, which is somewhat consistent with the stronger inverse association previously reported for distal compared to proximal colon cancer diagnosed at any age [68]. In addition, although participants were asked to report previous supplement use as $>2$ years before questionnaire completion, the association between antacid use (indicated for heartburn/ingestion or bloating) and increased risk of proximal colon, but not distal colon/rectal cancer, may be partly explained by subsite-specific early symptoms for EOCRC [69].

Beyond diet, antibiotics are also known to influence the gut microbiome [70,71], which has been implicated in CRC carcinogenesis, possibly through bacterial involvement in nutrient metabolism and direct interaction with gut mucosa [72]. There is concern regarding the increasing prescription of antibiotics in past decades, especially among children and youth, and their possible link to EO-CRC [11, 12, 25]. Recent epidemiological evidence suggests an association between antibiotic use and overall CRC risk, with potential differences by antibiotic type and anatomical subsite [73-76]. Our finding of a lower EO-CRC risk for antibiotic use restricted to childhood and a non-significant increase in risk for use during both childhood and adulthood possibly suggests that use over longer periods may confer increased risk [74]; however, further evaluation with more detailed data (e.g., duration, number of prescriptions) is required.

Another notable epidemiologic shift in recent birth cohorts is the increase in CT scan exposure among pediatric populations [26]. Thus, the suggestive association between having more $\mathrm{CT}$ scans (i.e., $\geq 3$ ) and increased EO-CRC risk observed in our study deserves attention in future studies. 
Table 3 Associations between lifestyle factors and risk of early-onset colorectal cancer, Ontario, Canada, 2018-2019

\begin{tabular}{|c|c|c|c|c|}
\hline \multirow[t]{2}{*}{ Variables } & \multirow{2}{*}{$\begin{array}{l}\begin{array}{l}\text { Cases } \\
(n=175)\end{array} \\
n^{\mathrm{a}} \\
(\%)\end{array}$} & \multirow{2}{*}{$\begin{array}{l}\begin{array}{l}\text { Controls } \\
(n=253)\end{array} \\
n^{\mathrm{a}} \\
(\%)\end{array}$} & \multirow[t]{2}{*}{$\begin{array}{l}\text { Age- and sex-adjusted } \\
\text { OR }^{\text {b }}(95 \% \mathrm{CI})\end{array}$} & \multirow{2}{*}{$\begin{array}{l}\text { Multivariable- } \\
\text { adjusted } \mathrm{OR}^{\mathrm{c}}(95 \% \\
\mathrm{CI})\end{array}$} \\
\hline & & & & \\
\hline \multicolumn{5}{|l|}{ Smoking status ${ }^{\mathrm{d}}$} \\
\hline Never smoker & $\begin{array}{l}110 \\
(63)\end{array}$ & $\begin{array}{l}172 \\
(68)\end{array}$ & 1.00 & 1.00 \\
\hline Ever smoker & $\begin{array}{l}65 \\
(37)\end{array}$ & $\begin{array}{l}81 \\
(32)\end{array}$ & $1.13(0.75-1.72)$ & $1.07(0.68-1.67)$ \\
\hline \multicolumn{5}{|l|}{ Pack-years of smoking } \\
\hline Tertile $1(<3.5)$ & $\begin{array}{l}33 \\
(19)\end{array}$ & $\begin{array}{l}27 \\
(11)\end{array}$ & $1.77(1.00-3.16)$ & $1.94(1.04-3.60)$ \\
\hline Tertile 2 (3.5-9.9) & $\begin{array}{l}12 \\
(7)\end{array}$ & $\begin{array}{l}27 \\
(11)\end{array}$ & $0.69(0.33-1.42)$ & $0.60(0.27-1.31)$ \\
\hline Tertile $3(\geq 10.0)$ & $\begin{array}{l}20 \\
(11)\end{array}$ & $\begin{array}{l}27 \\
(11)\end{array}$ & $0.96(0.50-1.81)$ & $0.79(0.40-1.57)$ \\
\hline \multicolumn{5}{|l|}{ Secondhand smoke exposure $e^{e}$} \\
\hline \multicolumn{5}{|l|}{ Two years ago } \\
\hline Never & $\begin{array}{l}115 \\
(70)\end{array}$ & $\begin{array}{l}167 \\
(70)\end{array}$ & 1.00 & 1.00 \\
\hline$<2$ h/day & $\begin{array}{l}40 \\
(24)\end{array}$ & $\begin{array}{l}62 \\
(26)\end{array}$ & $1.17(0.72-1.91)$ & $1.15(0.69-1.92)$ \\
\hline$\geq 2$ h/day & $\begin{array}{l}9 \\
(5)\end{array}$ & $\begin{array}{l}9 \\
(4)\end{array}$ & $1.54(0.58-4.10)$ & $1.86(0.64-5.43)$ \\
\hline Unknown & 11 & 15 & & \\
\hline \multicolumn{5}{|l|}{ Childhood and teenage years } \\
\hline Never & $\begin{array}{l}44 \\
(28)\end{array}$ & $\begin{array}{l}89 \\
(38)\end{array}$ & 1.00 & 1.00 \\
\hline$<2$ h/day & $\begin{array}{l}58 \\
(36)\end{array}$ & $\begin{array}{l}80 \\
(34)\end{array}$ & $1.28(0.77-2.13)$ & $1.22(0.71-2.11)$ \\
\hline$\geq 2 \mathrm{~h} /$ day & $\begin{array}{l}58 \\
(36)\end{array}$ & $\begin{array}{l}67 \\
(28)\end{array}$ & $1.35(0.80-2.29)$ & $1.29(0.73-2.28)$ \\
\hline Unknown & 15 & 17 & & \\
\hline \multicolumn{5}{|c|}{ Alcohol consumption ${ }^{\mathrm{f}}$ (2 years ago) } \\
\hline Less than once per month & $\begin{array}{l}59 \\
(34)\end{array}$ & $\begin{array}{l}85 \\
(34)\end{array}$ & 1.00 & 1.00 \\
\hline $1-3$ times per month & $\begin{array}{l}39 \\
(22)\end{array}$ & $\begin{array}{l}61 \\
(24)\end{array}$ & $0.97(0.57-1.65)$ & $1.05(0.59-1.85)$ \\
\hline $1-6$ times per week & $\begin{array}{l}58 \\
(33)\end{array}$ & $\begin{array}{l}84 \\
(33)\end{array}$ & $1.06(0.65-1.73)$ & $1.01(0.60-1.70)$ \\
\hline Daily & $\begin{array}{l}19 \\
(11)\end{array}$ & $\begin{array}{l}23 \\
(9)\end{array}$ & $1.24(0.60-2.55)$ & $1.07(0.49-2.32)$ \\
\hline \multicolumn{5}{|c|}{ Physical activity ${ }^{\mathrm{g}}$ ( 2 years ago) } \\
\hline Active & $\begin{array}{l}78 \\
(45)\end{array}$ & $\begin{array}{l}130 \\
(51)\end{array}$ & 1.00 & 1.00 \\
\hline Somewhat active & $\begin{array}{l}34 \\
(19)\end{array}$ & $\begin{array}{l}48 \\
(19)\end{array}$ & $1.14(0.67-1.94)$ & $1.16(0.66-2.02)$ \\
\hline Insufficiently active & $\begin{array}{l}63 \\
(36)\end{array}$ & $\begin{array}{l}75 \\
(30)\end{array}$ & $1.33(0.85-2.08)$ & $1.46(0.90-2.38)$ \\
\hline \multicolumn{5}{|l|}{ Sedentary time ${ }^{\mathrm{h}}$ ( 2 years ago) } \\
\hline$<5 \mathrm{~h} /$ day & $\begin{array}{l}41 \\
(23)\end{array}$ & $\begin{array}{l}73 \\
(29)\end{array}$ & 1.00 & 1.00 \\
\hline 5 to $<10 \mathrm{~h} /$ day & $\begin{array}{l}93 \\
(53)\end{array}$ & $\begin{array}{l}140 \\
(55)\end{array}$ & $1.20(0.75-1.93)$ & $1.23(0.75-2.03)$ \\
\hline \multirow[t]{2}{*}{$\geq 10 \mathrm{~h} /$ day } & $\begin{array}{l}41 \\
(23)\end{array}$ & $\begin{array}{l}40 \\
(16)\end{array}$ & $1.97(1.09-3.59)$ & $1.93(1.02-3.65)$ \\
\hline & & & $p_{\text {trend }}{ }^{\mathrm{i}}=0.03$ & $p_{\text {trend }} \mathrm{i}=0.049$ \\
\hline \multicolumn{5}{|l|}{ Body mass index ${ }^{\mathrm{j}}$} \\
\hline Two years ago & & & & \\
\hline
\end{tabular}


Table 3 (continued)

\begin{tabular}{|c|c|c|c|c|}
\hline \multirow[t]{2}{*}{ Variables } & \multirow{2}{*}{$\begin{array}{l}\begin{array}{l}\text { Cases } \\
(n=175)\end{array} \\
n^{\mathrm{a}} \\
(\%)\end{array}$} & \multirow{2}{*}{$\begin{array}{l}\begin{array}{l}\text { Controls } \\
(n=253)\end{array} \\
n^{\mathrm{a}} \\
(\%)\end{array}$} & \multirow[t]{2}{*}{$\begin{array}{l}\text { Age- and sex-adjusted } \\
\text { OR }^{\text {b }}(95 \% \text { CI })\end{array}$} & \multirow{2}{*}{$\begin{array}{l}\text { Multivariable- } \\
\text { adjusted OR }{ }^{\mathrm{c}}(95 \% \\
\text { CI) }\end{array}$} \\
\hline & & & & \\
\hline Normal/underweight & $\begin{array}{l}79 \\
(45)\end{array}$ & $\begin{array}{l}100 \\
(40)\end{array}$ & 1.00 & 1.00 \\
\hline Overweight & $\begin{array}{l}52 \\
(30)\end{array}$ & $\begin{array}{l}85 \\
(34)\end{array}$ & $0.72(0.45-1.15)$ & $0.57(0.34-0.94)$ \\
\hline \multirow[t]{2}{*}{ Obese } & $\begin{array}{l}44 \\
(25)\end{array}$ & $\begin{array}{l}67 \\
(27)\end{array}$ & $0.74(0.45-1.22)$ & $0.59(0.34-1.01)$ \\
\hline & & & $p_{\text {trend }}{ }^{\mathrm{i}}=0.26$ & $p_{\text {trend }}{ }^{\mathrm{i}}=0.06$ \\
\hline \multicolumn{5}{|l|}{ Early age 20 s } \\
\hline Normal/underweight & $\begin{array}{l}124 \\
(71)\end{array}$ & $\begin{array}{l}164 \\
(65)\end{array}$ & 1.00 & 1.00 \\
\hline Overweight & $\begin{array}{l}39 \\
(22)\end{array}$ & $\begin{array}{l}53 \\
(21)\end{array}$ & $1.07(0.65-1.77)$ & $1.06(0.63-1.80)$ \\
\hline \multirow[t]{2}{*}{ Obese } & $\begin{array}{l}12 \\
\text { (7) }\end{array}$ & $\begin{array}{l}54 \\
(14)\end{array}$ & $0.49(0.24-1.01)$ & $0.43(0.20-0.90)$ \\
\hline & & & $p_{\text {trend }}{ }^{\mathrm{i}}=0.10$ & $p_{\text {trend }}{ }^{\mathrm{i}}=0.06$ \\
\hline
\end{tabular}

$C I$ confidence interval; $O R$ odds ratio

aNumbers may not sum up to totals due to missing data. An "unknown" category is shown for variables with $>5 \%$ missing data

${ }^{\mathrm{b}}$ Adjusted for age (continuous, years; age at diagnosis for cases and at questionnaire completion for controls) and sex

${ }^{\mathrm{c}}$ Adjusted for age (continuous, years), sex, family history of CRC (no, yes, unknown), regular aspirin/ NSAID use (never/ever), smoking (never/ever), physical activity (active, somewhat active, insufficiently active), BMI (continuous, $\mathrm{kg} / \mathrm{m}^{2}$ ), alcohol consumption (< once/month, 1-3 times/month, 1-6 times/week, daily), red/processed meat intake (continuous, servings/week), total fruit and vegetable intake (continuous, servings/day), high-fiber food intake (continuous, servings/day), and calcium supplement use (never/ever)

${ }^{\mathrm{d}}$ Ever smoked $\geq 100$ cigarettes before 2 years ago. Pack-year was calculated by multiplying the number of packs of cigarettes smoked per day ( 1 pack $=20$ cigarettes) by the number of years smoked

${ }^{e}$ Duration of daily exposure to the tobacco smoke of others at home, work, or public places (averaged over weekdays and weekends)

${ }^{\mathrm{f}}$ Frequency of drinking alcoholic beverages (e.g., 12-oz can/bottle of beer, 4-oz glass of wine, 1.5-oz shot of hard liquor) 2 years ago

gefined based on a physical activity score derived using the Godin-Shephard Leisure-Time Physical Activity Questionnaire [27], where weekly frequency (times per week) of strenuous and moderate exercise ( 2 years ago) was multiplied by 9 and 5, respectively, and summed across activity types to calculate the composite score (active: $\geq 24$ units; somewhat active: 14-23 units; insufficiently active: $<14$ units)

${ }^{\mathrm{h}}$ Average number of hours per day spent sitting at work, at school, at home, in a car/bus/train, and during leisure time (e.g., watching TV, playing video games, using computer, reading, socializing) 2 years ago, calculated as $[(5 \times$ number of hours per day sitting on weekdays $)+(2 \times$ number of hours per day sitting on weekends) $] \div 7$

${ }^{\mathrm{i}} p$ value for linear trend calculated by treating the ordinal variable or the median value of each category (where applicable) as a continuous variable in the model, shown only when $p_{\text {trend }}$ is $<0.10$ for at least one of the age- and sex- adjusted and multivariable-adjusted ORs

${ }^{\mathrm{j}}$ Calculated by dividing weight $(\mathrm{kg})$ by height $(\mathrm{m})$ squared and classified as normal/underweight $(<25.0 \mathrm{~kg} /$ $\mathrm{m}^{2}$ ), overweight $\left(25.0-29.9 \mathrm{~kg} / \mathrm{m}^{2}\right)$, or obese $\left(\geq 30.0 \mathrm{~kg} / \mathrm{m}^{2}\right)$

Previously, a small case-control study reported an association between pelvic irradiation and risk of advanced colorectal neoplasm among young adults [77], and an Australian cohort study reported excess risks of gastrointestinal malignancies among persons exposed to a greater number of CT scans during childhood or adolescence [78]. Notably, the increased risk associated with having 3 or more CT scans but a lower risk with only 1 or 2 scans in our study may be indicative of a non-linear dose-response effect frequently seen for low-dose ionizing radiation $[79,80]$, although further investigation is needed given the relatively small numbers and absence of information on indication of CT scan.

Allergic conditions have been associated with reduced risks of certain cancers [81]; however, the association with 
Table 4 Associations between dietary factors and risk of early-onset colorectal cancer, Ontario, Canada, 2018-2019

\begin{tabular}{|c|c|c|c|c|}
\hline \multirow[t]{2}{*}{ Variables } & $\begin{array}{l}\text { Cases } \\
(n=175)\end{array}$ & $\begin{array}{l}\text { Controls } \\
(n=253)\end{array}$ & \multirow[t]{2}{*}{$\begin{array}{l}\text { Age- and sex-adjusted } \\
\text { OR }^{\text {a }}(95 \% \mathrm{CI})\end{array}$} & \multirow{2}{*}{$\begin{array}{l}\text { Multivariable- } \\
\text { adjusted OR } \\
(95 \% \mathrm{CI})\end{array}$} \\
\hline & $\begin{array}{l}n \\
(\%)\end{array}$ & $\begin{array}{l}n \\
(\%)\end{array}$ & & \\
\hline
\end{tabular}

Dietary intake 2 years ago ${ }^{\mathrm{c}}$

Total fruits and vegetables, servings/day

$\begin{array}{lll}<3 & 69 & 85 \\ 3 \text { to }<6 & (39) & (34) \\ & 76 & 111 \\ \geq 6 & (43) & (44) \\ & 30 & 57 \\ & (17) & (23)\end{array}$

Fruits, servings/day ${ }^{\mathrm{e}}$

1 to $<3 \quad 2$

Vegetables, servings/day ${ }^{\mathrm{f}}$

1 to $<3$

High-fiber foods, servings/day ${ }^{\mathrm{g}}$

$<$ to $<3$
$\geq 3$

69

(39)

74

(42)

32

(18)

Red meat, servings/week ${ }^{\mathrm{h}}$

$\begin{array}{ll}<2 & 29 \\ & (17) \\ 2-4 & 78 \\ & (45) \\ & 68 \\ & 68\end{array}$

Processed meat, servings/week ${ }^{\mathrm{h}}$

$\begin{array}{ll}<1 & 21 \\ & (12) \\ 1-2 & 69 \\ & (39) \\ & \\ & 85 \\ & \end{array}$

Sugary drinks, drinks/week ${ }^{\mathrm{i}}$

$\begin{array}{ll}<1 & 39 \\ & (22) \\ 1-6 & 89\end{array}$

$44 \quad 61$

(25) (24)

$95 \quad 138$

(54) (55)

$\begin{array}{ll}1.00 & 1.00 \\ 0.69(0.43-1.08) & 0.76(0.46-1.25) \\ 0.53(0.30-0.96) & 0.58(0.30-1.13) \\ p_{\text {trend }}{ }^{\mathrm{d}}=0.03 & p_{\text {trend }}{ }^{\mathrm{d}}=0.11\end{array}$

$1.00 \quad 1.00$

$0.93(0.57-1.51) \quad 0.96(0.57-1.60)$

$0.84(0.46-1.53) \quad 0.95(0.49-1.85)$

$1.00 \quad 1.00$

$0.61(0.35-1.09) \quad 0.56(0.30-1.03)$

$0.50(0.26-0.93) \quad 0.52(0.26-1.07)$

$p_{\text {trend }}{ }^{\mathrm{d}}=0.03 \quad p_{\text {trend }}{ }^{\mathrm{d}}=0.08$

$1.00 \quad 1.00$

$0.90(0.58-1.39) \quad 1.27(0.77-2.09)$

$0.94(0.53-1.66) \quad 1.45(0.75-2.80)$

(18)

45

(18)

121

(48)

87

(34)

$21 \quad 39$

(12) (15)

(39) 108

$85-106$

(49) $\quad(42)$

39

(34)

123

(49)
$1.00 \quad 1.00$

$0.92(0.52-1.61) \quad 0.82(0.45-1.51)$

$1.11(0.62-1.99) \quad 1.06(0.56-1.98)$

$1.00 \quad 1.00$

$1.09(0.58-2.04) \quad 0.96(0.49-1.88)$

$1.41(0.76-2.63) \quad 1.23(0.62-2.42)$

$1.00 \quad 1.00$

$1.58(0.98-2.55) \quad 1.86(1.11-3.13)$ 
Table 4 (continued)

\begin{tabular}{|c|c|c|c|c|}
\hline \multirow[t]{2}{*}{ Variables } & \multirow{2}{*}{$\begin{array}{l}\text { Cases } \\
(n=175) \\
n \\
(\%)\end{array}$} & \multirow{2}{*}{$\begin{array}{l}\text { Controls } \\
(n=253)\end{array}$} & \multirow[t]{2}{*}{$\begin{array}{l}\text { Age- and sex-adjusted } \\
\mathrm{OR}^{\mathrm{a}}(95 \% \mathrm{CI})\end{array}$} & \multirow{2}{*}{$\begin{array}{l}\text { Multivariable- } \\
\text { adjusted OR } \\
(95 \% \text { CI })\end{array}$} \\
\hline & & & & \\
\hline \multirow[t]{2}{*}{$\geq 7$} & $\begin{array}{l}47 \\
(27)\end{array}$ & $\begin{array}{l}44 \\
(17)\end{array}$ & $2.53(1.41-4.51)$ & $2.99(1.57-5.68)$ \\
\hline & & & $p_{\text {trend }}{ }^{\mathrm{d}}=0.003$ & $p_{\text {trend }}{ }^{\mathrm{d}}=0.002$ \\
\hline \multicolumn{5}{|c|}{ Sugary desserts, times/week ${ }^{\mathrm{j}}$} \\
\hline$<3$ & $\begin{array}{l}37 \\
(21)\end{array}$ & $\begin{array}{l}74 \\
(29)\end{array}$ & 1.00 & 1.00 \\
\hline $3-6$ & $\begin{array}{l}64 \\
(37)\end{array}$ & $\begin{array}{l}68 \\
(27)\end{array}$ & $1.89(1.11-3.23)$ & $2.28(1.28-4.04)$ \\
\hline$\geq 7$ & $\begin{array}{l}74 \\
(42)\end{array}$ & $\begin{array}{l}111 \\
(44)\end{array}$ & $1.42(0.86-2.35)$ & $1.45(0.86-2.47)$ \\
\hline \multicolumn{5}{|c|}{ Fast food, times/week ${ }^{\mathrm{k}}$} \\
\hline$<1$ & $\begin{array}{l}26 \\
(15)\end{array}$ & $\begin{array}{l}55 \\
(22)\end{array}$ & 1.00 & 1.00 \\
\hline 1 & $\begin{array}{l}71 \\
(41)\end{array}$ & $\begin{array}{l}99 \\
(39)\end{array}$ & $1.57(0.89-2.77)$ & $1.55(0.85-2.81)$ \\
\hline$\geq 2$ & $\begin{array}{l}78 \\
(45)\end{array}$ & $\begin{array}{l}99 \\
(39)\end{array}$ & $1.84(1.03-3.28)$ & $1.84(0.98-3.46)$ \\
\hline & \multicolumn{3}{|c|}{ Canned food, times/week ${ }^{1}$} & $p_{\text {trend }}{ }^{\mathrm{d}}=0.07$ \\
\hline$<1$ & $\begin{array}{l}33 \\
(19)\end{array}$ & $\begin{array}{l}63 \\
(25)\end{array}$ & 1.00 & 1.00 \\
\hline $1-2$ & $\begin{array}{l}78 \\
(45)\end{array}$ & $\begin{array}{l}105 \\
(42)\end{array}$ & $1.34(0.79-2.27)$ & $1.49(0.85-2.61)$ \\
\hline$\geq 3$ & $\begin{array}{l}64 \\
(37)\end{array}$ & $\begin{array}{l}85 \\
(34)\end{array}$ & $1.48(0.86-2.54)$ & $1.70(0.95-3.05)$ \\
\hline & & & $p_{\text {trend }}{ }^{\mathrm{d}}=0.18$ & $p_{\text {trend }}{ }^{\mathrm{d}}=0.09$ \\
\hline \multicolumn{5}{|c|}{ Processed snacks, times/day ${ }^{\mathrm{m}}$} \\
\hline$<1$ & $\begin{array}{l}116 \\
(66)\end{array}$ & $\begin{array}{l}183 \\
(72)\end{array}$ & 1.00 & 1.00 \\
\hline 1 to $<2$ & $\begin{array}{l}39 \\
(22)\end{array}$ & $\begin{array}{l}48 \\
(19)\end{array}$ & $1.34(0.82-2.19)$ & $1.33(0.79-2.25)$ \\
\hline$\geq 2$ & $\begin{array}{l}20 \\
(11)\end{array}$ & $\begin{array}{l}22 \\
(9)\end{array}$ & $1.43(0.74-2.79)$ & $1.55(0.76-3.15)$ \\
\hline \multicolumn{5}{|c|}{ Coffee or tea, cups/day ${ }^{n}$} \\
\hline$<1$ & $\begin{array}{l}27 \\
(15)\end{array}$ & $\begin{array}{l}55 \\
(22)\end{array}$ & 1.00 & 1.00 \\
\hline 1 to $<3$ & $\begin{array}{l}99 \\
(57)\end{array}$ & $\begin{array}{l}142 \\
(56)\end{array}$ & $1.34(0.78-2.31)$ & $1.43(0.80-2.54)$ \\
\hline$\geq 3$ & $\begin{array}{l}49 \\
(28)\end{array}$ & $\begin{array}{l}56 \\
(22)\end{array}$ & $1.43(0.77-2.66)$ & $1.68(0.85-3.30)$ \\
\hline \multicolumn{5}{|c|}{ Water, glasses/day } \\
\hline$<3$ & $\begin{array}{l}59 \\
(34)\end{array}$ & $\begin{array}{l}75 \\
(30)\end{array}$ & 1.00 & 1.00 \\
\hline 3 to $<8$ & $\begin{array}{l}94 \\
(54)\end{array}$ & $\begin{array}{l}136 \\
(54)\end{array}$ & $0.96(0.62-1.48)$ & $1.05(0.65-1.68)$ \\
\hline$\geq 8$ & $\begin{array}{l}22 \\
(13)\end{array}$ & $\begin{array}{l}42 \\
(17)\end{array}$ & $0.76(0.40-1.45)$ & $0.85(0.42-1.72)$ \\
\hline \multicolumn{5}{|c|}{ Artificial sweeteners, times/week } \\
\hline$<1$ & $\begin{array}{l}116 \\
(66)\end{array}$ & $\begin{array}{l}173 \\
(68)\end{array}$ & 1.00 & 1.00 \\
\hline
\end{tabular}


Table 4 (continued)

\begin{tabular}{|c|c|c|c|c|}
\hline \multirow[t]{2}{*}{ Variables } & \multirow{2}{*}{$\begin{array}{l}\text { Cases } \\
(n=175) \\
n \\
(\%)\end{array}$} & \multirow{2}{*}{$\begin{array}{l}\text { Controls } \\
(n=253) \\
n \\
(\%)\end{array}$} & \multirow[t]{2}{*}{$\begin{array}{l}\text { Age- and sex-adjusted } \\
\mathrm{OR}^{\mathrm{a}}(95 \% \mathrm{CI})\end{array}$} & \multirow{2}{*}{$\begin{array}{l}\text { Multivariable- } \\
\text { adjusted OR } \\
(95 \% \text { CI })\end{array}$} \\
\hline & & & & \\
\hline $1-6$ & $\begin{array}{l}31 \\
(18)\end{array}$ & $\begin{array}{l}53 \\
(21)\end{array}$ & $0.88(0.53-1.47)$ & $1.19(0.68-2.08)$ \\
\hline$\geq 7$ & $\begin{array}{l}28 \\
(16)\end{array}$ & $\begin{array}{l}27 \\
(11)\end{array}$ & $1.46(0.81-2.63)$ & $1.66(0.89-3.13)$ \\
\hline \multicolumn{5}{|l|}{ Agave syrup, times/week } \\
\hline$<1$ & $\begin{array}{l}166 \\
(95)\end{array}$ & $\begin{array}{l}226 \\
(89)\end{array}$ & 1.00 & 1.00 \\
\hline$\geq 1$ & $\begin{array}{l}9 \\
(5)\end{array}$ & $\begin{array}{l}27 \\
(11)\end{array}$ & $0.41(0.19-0.92)$ & $0.45(0.19-1.04)$ \\
\hline \multicolumn{5}{|l|}{ Western-like dietary pattern score ${ }^{o}$} \\
\hline Quartile $1(0-9)$ & $\begin{array}{l}32 \\
(18)\end{array}$ & $\begin{array}{l}65 \\
(26)\end{array}$ & 1.00 & 1.00 \\
\hline Quartile $2(10-13)$ & $\begin{array}{l}46 \\
(26)\end{array}$ & $\begin{array}{l}67 \\
(26)\end{array}$ & $1.33(0.75-2.38)$ & $1.46(0.80-2.67)$ \\
\hline Quartile 3 (14-17) & $\begin{array}{l}45 \\
(26)\end{array}$ & $\begin{array}{l}60 \\
(24)\end{array}$ & $1.58(0.87-2.85)$ & $1.62(0.87-3.04)$ \\
\hline Quartile 4 (18-27) & $\begin{array}{l}52 \\
(30)\end{array}$ & $\begin{array}{l}61 \\
(24)\end{array}$ & $1.95(1.07-3.56)$ & $1.92(1.01-3.66)$ \\
\hline & & & $p_{\text {trend }}{ }^{\mathrm{d}}=0.03$ & $p_{\text {trend }}{ }^{\mathrm{d}}=0.047$ \\
\hline \multicolumn{5}{|l|}{ Supplement use before 2 years ago ${ }^{\mathrm{p}}$} \\
\hline \multicolumn{5}{|l|}{ Calcium supplement } \\
\hline Never & $\begin{array}{l}145 \\
(83)\end{array}$ & $\begin{array}{l}193 \\
(76)\end{array}$ & 1.00 & 1.00 \\
\hline Ever & $\begin{array}{l}30 \\
(17)\end{array}$ & $\begin{array}{l}60 \\
(24)\end{array}$ & $0.58(0.35-0.98)$ & $0.53(0.31-0.92)$ \\
\hline \multicolumn{5}{|l|}{ Antacid } \\
\hline Never & $\begin{array}{l}111 \\
(63)\end{array}$ & $\begin{array}{l}153 \\
(60)\end{array}$ & 1.00 & 1.00 \\
\hline Ever & $\begin{array}{l}64 \\
(37)\end{array}$ & $\begin{array}{l}100 \\
(40)\end{array}$ & $0.83(0.55-1.25)$ & $0.87(0.56-1.36)$ \\
\hline \multicolumn{5}{|l|}{ Vitamin D/cod liver oil supplement } \\
\hline Never & $\begin{array}{l}114 \\
(65)\end{array}$ & $\begin{array}{l}146 \\
(58)\end{array}$ & 1.00 & 1.00 \\
\hline Ever & $\begin{array}{l}61 \\
(35)\end{array}$ & $\begin{array}{l}107 \\
(42)\end{array}$ & $0.69(0.45-1.05)$ & $0.83(0.52-1.31)$ \\
\hline \multicolumn{5}{|l|}{ Prebiotic fiber supplement } \\
\hline Never & $\begin{array}{l}138 \\
(79)\end{array}$ & $\begin{array}{l}196 \\
(77)\end{array}$ & 1.00 & 1.00 \\
\hline Ever & $\begin{array}{l}37 \\
(21)\end{array}$ & $\begin{array}{l}57 \\
(23)\end{array}$ & $0.84(0.52-1.37)$ & $1.05(0.62-1.80)$ \\
\hline \multicolumn{5}{|l|}{ Probiotic supplement } \\
\hline Never & $\begin{array}{l}143 \\
(82)\end{array}$ & $\begin{array}{l}203 \\
(80)\end{array}$ & 1.00 & 1.00 \\
\hline Ever & $\begin{array}{l}32 \\
(18)\end{array}$ & $\begin{array}{l}50 \\
(20)\end{array}$ & $0.91(0.54-1.51)$ & $1.09(0.63-1.89)$ \\
\hline Folic acid supplement (females only) & $(n=101)$ & $(n=141)$ & & \\
\hline No & $\begin{array}{l}29 \\
(29)\end{array}$ & $\begin{array}{l}43 \\
(30)\end{array}$ & 1.00 & 1.00 \\
\hline Yes & $\begin{array}{l}72 \\
(71)\end{array}$ & $\begin{array}{l}98 \\
(70)\end{array}$ & $0.72(0.38-1.35)$ & $0.78(0.40-1.52)$ \\
\hline
\end{tabular}


Table 4 (continued)

$C I$ confidence interval; $O R$ odds ratio

${ }^{\mathrm{a}}$ Adjusted for age (continuous, years; age at diagnosis for cases and at questionnaire completion for controls) and sex

${ }^{\mathrm{b}}$ Adjusted for age (continuous, years), sex, family history of CRC (no, yes, unknown), regular aspirin/NSAID use (never/ever), smoking (never/ ever), physical activity (active, somewhat active, insufficiently active), BMI (continuous, $\mathrm{kg} / \mathrm{m}^{2}$ ), alcohol consumption $(<$ once/month, $1-3$ times/month, 1-6 times/week, daily), red/processed meat intake (continuous, servings/week), total fruit and vegetable intake (continuous, servings/day), high-fiber food intake (continuous, servings/day), and calcium supplement use (never/ever)

${ }^{\mathrm{c}}$ All food and beverage variables are based on usual consumption 2 years before questionnaire completion

${ }^{\mathrm{d}} p$ value for linear trend calculated by treating the median value of each category as a continuous variable in the model, shown only when $p_{\text {trend }}$ is $<0.10$ for at least one of the age- and sex-adjusted and multivariable-adjusted ORs

${ }^{\mathrm{e}}$ Examples for one serving of fruit: 1 medium-sized fresh fruit, $1 / 2$ cup of chopped, cooked, or canned fruit, $1 / 4$ cup of dried fruit, $1 / 2$ cup of fruit juice

${ }^{\mathrm{f}}$ Examples for one serving of vegetables: 1 cup of raw leafy vegetables, 1/2 cup of other vegetables (cooked, canned, frozen, or chopped raw), $1 / 2$ cup of vegetable juice

${ }^{\mathrm{g}}$ Foods high in fiber, such as wholegrain bread (not white bread), wholegrain or high-fiber breakfast cereal/muesli/bran, brown rice, barley, oats, and legumes (beans, peas, lentils). Examples of one serving of high-fiber food: 1 slice of wholegrain bread, 1/2 cup of cooked or cold high-fiber cereals, $1 / 2$ cup of brown rice or wholegrains

${ }^{\mathrm{h}}$ One serving of red/processed meat defined as $2-3 \mathrm{oz}$ or the size of the palm of hand

${ }^{i}$ Sugary drinks such as soft drinks (excluding diet soda), vitamin drinks, energy drinks, and specialty coffee with syrup (e.g., mocha)

${ }^{\mathrm{j}}$ Desserts containing sugar, such as candy, chocolate bars, cake, cookies, and ice cream

${ }^{\mathrm{k}}$ Includes foods from fast food restaurants (e.g., burger, fries, taco), pizza, and instant meals (e.g., instant ramen noodles)

${ }^{1}$ Any canned foods (e.g., canned corn, canned fruit, canned tomato sauce)

${ }^{\mathrm{m}}$ Any processed snack foods such as chips, crackers, white bread, and sugary cereals

${ }^{\mathrm{n}}$ Includes both caffeinated and decaffeinated coffee or tea

${ }^{\circ}$ Composite dietary score derived based on six non-beneficial (red meat, processed meat, sugary drinks, sugary desserts, fast food, and processed snacks) and three beneficial (fruits, vegetables, and high-fiber foods) components. For each non-beneficial component, subjects in the first, second, third, and fourth quartile of intake were assigned a value of $0,1,2$, and 3, respectively; for each beneficial component, quartiles were reverse coded (i.e., $3,2,1$, and 0 , respectively). The final score was calculated by summing up values across all nine components, with higher scores indicating a more Westernized dietary pattern. The multivariable-adjusted OR was adjusted for all variables listed in footnote b, except for red/processed meat, total fruit and vegetable, and high-fiber food intake

${ }^{\mathrm{p}}$ Ever used the supplement regularly (at least once per week for at least one month) before 2 years ago

CRC remains inconsistent [82-84]. Our study found an association between history of allergy/asthma, especially earlier age at onset (and hence longer duration since onset), and reduced EO-CRC risk. Underlying mechanisms are unclear but may involve enhanced tumor immunosurveillance and immunoglobulin E-mediated immune responses against colorectal neoplasia [82, 85].

Evidence regarding the associations of reproductive factors with CRC risk is inconclusive [86-88], with the exception of exogenous hormones (OC and hormone replacement therapy [HRT]) as potential protective factors $[89,90]$. While OC use was not associated with EO-CRC risk in our study (and HRT was not assessed due to small numbers in this largely premenopausal sample), the reduced risk associated with parity may be partly explained by changes in endogenous sex hormones (e.g., estrogen, prolactin) during pregnancy [91] and warrants additional investigation according to menopausal status.

This study is one of the very few to investigate risk factors for EO-CRC and is the most comprehensive one to date. Strengths of the study included the population-based design, assessment of a wide range of traditional CRC risk factors, as well as novel medical, lifestyle, and dietary factors, and use of a web-based questionnaire to facilitate quality control of data collection. Our study also had several limitations. First, low response rates may have introduced selection bias; however, participating cases were not markedly different from eligible non-participating EO-CRC cases identified from the OCR in terms of age, stage, and cancer subsite. Participating controls also had similar distributions of major lifestyle factors (e.g., smoking, obesity) compared to those of the general young adult population in Ontario [92]. Second, survivor bias is possible though unlikely since most cases were recruited within several months of diagnosis. Additionally, the possibility of recall bias, measurement error, and type I error could not be ruled out. Finally, due to the relatively small sample size, our study may have been underpowered to detect modest associations, and sex- and subsite-specific analyses should be interpreted with caution.

In conclusion, this study provides novel findings on a range of factors possibly associated with EO-CRC risk. Several modifiable factors, particularly sedentary behavior and unhealthy diet characterized by sugary drinks and fast food, emerged as potential risk factors for EO-CRC, while calcium 
supplement use may be associated with reduced risk. Findings from this study represent early steps in understanding the etiology of CRC among younger persons and warrant confirmation in large prospective studies with long follow-up so that prevention and screening strategies may be targeted at this subpopulation.

Supplementary Information The online version contains supplementary material available at https://doi.org/10.1007/s10552-021-01456-8.

Acknowledgments This research was supported by an Innovation Grant from the Canadian Cancer Society in partnership with the Canadian Institutes of Health Research-Institute for Cancer Research (CCS grant \#705559/CIHR-ICR grant \#155424 to M. Cotterchio). Parts of this material are based on data and information provided by Ontario Health (Cancer Care Ontario). The opinions, reviews, views and conclusions reported in this publication are those of the authors and do not necessarily reflect those of Ontario Health (Cancer Care Ontario). No endorsement by Ontario Health (Cancer Care Ontario) is intended or should be inferred. The authors thank Alice Peter for her contribution to the inception of this study, and David Northrup and staff at the Institute for Social Research, York University for services provided in control recruitment.

Funding This research was supported by an Innovation Grant from the Canadian Cancer Society in partnership with the Canadian Institutes of Health Research-Institute for Cancer Research (CCS grant \#705559/ CIHR-ICR grant \#155424 to M. Cotterchio).

Data availability The data described in this manuscript are not publicly available due to privacy and ethical restrictions.

Code availability Available upon reasonable request.

\section{Declarations}

Conflict of interest The authors have no conflicts of interest to declare.

Ethical approval Ethics approval for this study was obtained from the University of Toronto Health Sciences Research Ethics Board.

Consent to participate Informed consent was obtained in order to allow the study to invite cancer cases to participate and implied consent was assumed from all individual participants (cases and controls) who completed the online study questionnaire.

Open Access This article is licensed under a Creative Commons Attribution 4.0 International License, which permits use, sharing, adaptation, distribution and reproduction in any medium or format, as long as you give appropriate credit to the original author(s) and the source, provide a link to the Creative Commons licence, and indicate if changes were made. The images or other third party material in this article are included in the article's Creative Commons licence, unless indicated otherwise in a credit line to the material. If material is not included in the article's Creative Commons licence and your intended use is not permitted by statutory regulation or exceeds the permitted use, you will need to obtain permission directly from the copyright holder. To view a copy of this licence, visit http://creativecommons.org/licenses/by/4.0/.

\section{References}

1. Sung H, Ferlay J, Siegel RL, Laversanne M, Soerjomataram I, Jemal A, Bray F (2021) Global cancer statistics 2020: GLOBOCAN estimates of incidence and mortality worldwide for 36 cancers in 185 countries. CA Cancer J Clin. https://doi.org/10.3322/ caac. 21660

2. Saad El Din K, Loree JM, Sayre EC, Gill S, Brown CJ, Dau H, De Vera MA (2020) Trends in the epidemiology of young-onset colorectal cancer: a worldwide systematic review. BMC Cancer 20(1):288. https://doi.org/10.1186/s12885-020-06766-9

3. O'Sullivan DE, Hilsden RJ, Ruan Y, Forbes N, Heitman SJ, Brenner DR (2020) The incidence of young-onset colorectal cancer in Canada continues to increase. Cancer Epidemiol 69:101828. https://doi.org/10.1016/j.canep.2020.101828

4. Siegel RL, Miller KD, Godin Sauer A, Fedewa SA, Butterly LF, Anderson JC, Cercek A, Smith PA, Jemal A (2020) Colorectal cancer statistics, 2020. CA Cancer J Clin 70(3):145-164. https:// doi.org/10.3322/caac. 21601

5. Feletto E, Yu XQ, Lew JB, St John DJB, Jenkins MA, Macrae FA, Mahady SE, Canfell K (2019) Trends in colon and rectal cancer incidence in Australia from 1982 to 2014: analysis of data on over 375,000 cases. Cancer Epidemiol Biomark Prev 28(1):83-90. https://doi.org/10.1158/1055-9965.EPI-18-0523

6. Vuik FER, Nieuwenburg SAV, Bardou M, Lansdorp-Vogelaar I, Dinis-Ribeiro M, Bento MJ, Zadnik V, Pellisé M, Esteban L, Kaminski MF, Suchanek S, Ngo O, Májek O, Leja M, Kuipers EJ, Spaander MCW (2019) Increasing incidence of colorectal cancer in young adults in Europe over the last 25 years. Gut 68(10):1820 1826. https://doi.org/10.1136/gutjnl-2018-317592

7. Siegel RL, Torre LA, Soerjomataram I, Hayes RB, Bray F, Weber TK, Jemal A (2019) Global patterns and trends in colorectal cancer incidence in young adults. Gut 68(12):2179-2185. https://doi. org/10.1136/gutjnl-2019-319511

8. Sung JJY, Chiu HM, Jung KW, Jun JK, Sekiguchi M, Matsuda T, Kyaw MH (2019) Increasing trend in young-onset colorectal cancer in Asia: more cancers in men and more rectal cancers. Am J Gastroenterol 114(2):322-329. https://doi.org/10.14309/ajg. 0000000000000133

9. Ahnen DJ, Wade SW, Jones WF, Sifri R, Mendoza Silveiras J, Greenamyer J, Guiffre S, Axilbund J, Spiegel A, You YN (2014) The increasing incidence of young-onset colorectal cancer: a call to action. Mayo Clin Proc 89(2):216-224. https://doi.org/10. 1016/j.mayocp.2013.09.006

10. Patel P, De P (2016) Trends in colorectal cancer incidence and related lifestyle risk factors in 15-49-year-olds in Canada, 19692010. Cancer Epidemiol 42:90-100. https://doi.org/10.1016/j. canep.2016.03.009

11. Stoffel EM, Murphy CC (2020) Epidemiology and mechanisms of the increasing incidence of colon and rectal cancers in young adults. Gastroenterology 158(2):341-353. https://doi.org/10. 1053/j.gastro.2019.07.055

12. Akimoto N, Ugai T, Zhong R, Hamada T, Fujiyoshi K, Giannakis M, Wu K, Cao Y, Ng K, Ogino S (2020) Rising incidence of earlyonset colorectal cancer-a call to action. Nat Rev Clin Oncol. https://doi.org/10.1038/s41571-020-00445-1

13. Yeo H, Betel D, Abelson JS, Zheng XE, Yantiss R, Shah MA (2017) Early-onset colorectal cancer is distinct from traditional colorectal cancer. Clin Coloretal Cancer 16(4):293-299.e6. https:// doi.org/10.1016/j.clcc.2017.06.002

14. Johnson CM, Wei C, Ensor JE, Smolenski DJ, Amos CI, Levin B, Berry DA (2013) Meta-analyses of colorectal cancer risk factors. Cancer Causes Control 24(6):1207-1222. https://doi.org/10.1007/ s10552-013-0201-5 
15. O'Sullivan DE, Sutherland RL, Town S, Chow K, Fan J, Forbes N, Heitman SJ, Hilsden RJ, Brenner DR (2021) Risk factors for early-onset colorectal cancer: a systematic review and meta-analysis. Clin Gastroenterol Hepatol. https://doi.org/10.1016/j.cgh. 2021.01.037

16. Rosato V, Bosetti C, Levi F, Polesel J, Zucchetto A, Negri E, La Vecchia C (2013) Risk factors for young-onset colorectal cancer. Cancer Causes Control 24(2):335-341. https://doi.org/10.1007/ s10552-012-0119-3

17. Liu PH, Wu K, Ng K, Zauber AG, Nguyen LH, Song M, He X, Fuchs CS, Ogino S, Willett WC, Chan AT, Giovannucci EL, Cao Y (2019) Association of obesity with risk of early-onset colorectal cancer among women. JAMA Oncol 5(1):37-44. https://doi.org/ 10.1001/jamaoncol.2018.4280

18. Nguyen LH, Liu PH, Zheng X, Keum N, Zong X, Li X, Wu K, Fuchs CS, Ogino S, Ng K, Willett WC, Chan AT, Giovannucci EL, Cao Y (2018) Sedentary behaviors, TV viewing time, and risk of young-onset colorectal cancer. JNCI Cancer Spectr 2(4):pky073. https://doi.org/10.1093/jncics/pky073

19. Kelty E, Ward SV, Cadby G, McCarthy NS, O'Leary P, Moses EK, Ee HC, Preen DB (2019) Familial and non-familial risk factors associated with incidence of colorectal cancer in young and middle-aged persons in Western Australia. Cancer Epidemiol 62:101591. https://doi.org/10.1016/j.canep.2019.101591

20. Syed AR, Thakkar P, Horne ZD, Abdul-Baki H, Kochhar G, Farah K, Thakkar S (2019) Old vs new: risk factors predicting early onset colorectal cancer. World J Gastrointest Oncol 11(11):10111020. https://doi.org/10.4251/wjgo.v11.i11.1011

21. Glover M, Mansoor E, Panhwar M, Parasa S, Cooper GS (2019) Epidemiology of colorectal cancer in average risk adults 20-39 years of age: a population-based national study. Dig Dis Sci 64(12):3602-3609. https://doi.org/10.1007/s10620-019-05690-8

22. Ali Khan U, Fallah M, Tian Y, Sundquist K, Sundquist J, Brenner H, Kharazmi E (2020) Personal history of diabetes as important as family history of colorecal cancer for risk of colorectal cancer: a nationwide cohort study. Am J Gastroenterol 115(7):1103-1109. https://doi.org/10.14309/ajg.0000000000000669

23. Gausman V, Dornblaser D, Anand S, Hayes RB, O'Connell K, Du M, Liang PS (2020) Risk factors associated with early-onset colorectal cancer. Clin Gastroenterol Hepatol 18(12):2752-2759. e2. https://doi.org/10.1016/j.cgh.2019.10.009

24. Low EE, Demb J, Liu L, Earles A, Bustamante R, Williams CD, Provenzale D, Kaltenbach T, Gawron AJ, Martinez ME, Gupta S (2020) Risk factors for early-onset colorectal cancer. Gastroenterology 159(2):492-501.e7. https://doi.org/10.1053/j.gastro.2020. 01.004

25. Hofseth LJ, Hebert JR, Chanda A, Chen H, Love BL, Pena MM, Murphy EA, Sajish M, Sheth A, Buckhaults PJ, Berger FG (2020) Early-onset colorectal cancer: initial clues and current views. Nat Rev Gastroenterol Hepatol 17(6):352-364. https://doi.org/10. 1038/s41575-019-0253-4

26. Miglioretti DL, Johnson E, Williams A, Greenlee RT, Weinmann S, Solberg LI, Feigelson HS, Roblin D, Flynn MJ, Vanneman N, Smith-Bindman R (2013) The use of computed tomography in pediatrics and the associated radiation exposure and estimated cancer risk. JAMA Pediatr 167(8):700-707. https://doi.org/10. 1001/jamapediatrics.2013.311

27. Godin G (2011) The Godin-Shephard Leisure-Time Physical Activity Questionnaire. Health Fit J Can 4(1):18-22. https://doi. org/10.14288/hfjc.v4i1.82

28. Amireault S, Godin G (2015) The Godin-Shephard leisure-time physical activity questionnaire: validity evidence supporting its use for classfying healthy adults into active and insufficiently active categories. Percept Mot Skills 120(2):604-622. https:// doi.org/10.2466/03.27.PMS.120v19x7
29. Steck SE, Murphy EA (2020) Dietary patterns and cancer risk. Nat Rev Cancer 20(2):125-138. https://doi.org/10.1038/ s41568-019-0227-4

30. World Cancer Research Fund/American Institute for Cancer Research (2018) Continuous Update Project Expert Report 2018. Diet, nutrition, physical activity and colorectal cancer. Available at http://www.dietandcancerreport.org. Accessed 12 Aug 2020

31. Peters RK, Garabrant DH, Yu MC, Mack TM (1989) A case-control study of occupatinal and dietary factors in colorectal cancer in young men by subsite. Cancer Res 49(19):5459-5468

32. Keum N, Giovannucci E (2019) Global burden of colorectal cancer: emerging trends, risk factors and prevention strategies. Nat Rev Gastroenterol Hepatol 16(12):713-732. https://doi.org/10. 1038/s41575-019-0189-8

33. Wei EK, Wolin KY, Colditz GA (2010) Time course of risk factors in cancer etiology and progression. J Clin Oncol 28(26):40524057. https://doi.org/10.1200/JCO.2009.26.9324

34. Jung YS, Ryu S, Chang Y, Yun KE, Park JH, Kim HJ, Cho YK, Sohn CI, Jeon WK, Kim BI, Park DI (2015) Risk factors for colorectal neoplasia in persons aged 30 to 39 years and 40 to 49 years. Gastrointest Endosc 81(3):637-645.e7. https://doi.org/10.1016/j. gie.2014.09.031

35. Kwak JY, Kim KM, Yang HJ, Yu KJ, Lee JG, Jeong YO, Shim SG (2016) Prevalence of colorectal adenomas in asymptomatic young adults: a window to early intervention? Scand J Gastroenterol 51(6):731-738. https://doi.org/10.3109/00365521.2015. 1130163

36. Lee SE, Jo HB, Kwack WG, Jeong YJ, Yoon YJ, Kang HW (2016) Characteristics of and risk factors for colorectal neoplasms in young adults in a screening population. World J Gastroenterol 22(10):2981-2992. https://doi.org/10.3748/wjg.v22.i10.2981

37. Kim JY, Jung YS, Park JH, Kim HJ, Cho YK, Sohn CI, Jeon WK, Kim BI, Choi KY, Park DI (2016) Different risk factors for advanced colorectal neoplasm in young adults. World J Gastroenterol 22(13):3611-3620. https://doi.org/10.3748/wjg.v22.i13. 3611

38. Kim NH, Jung YS, Yang HJ, Park SK, Park JH, Park DI, Sohn CI (2019) Prevalence of and risk factors for colorectal neoplasia in asymptomatic young adults (20-39 years old). Clin Gastroenterol Hepatol 17(1):115-122. https://doi.org/10.1016/j.cgh.2018.07.011

39. Breau G, Ellis U (2020) Risk factors associated with youngonset colorectal adenomas and cancer: a systematic review and meta-analysis of observational research. Cancer Control 27(1):1073274820976670. https://doi.org/10.1177/1073274820 976670

40. Umezawa S, Higurashi T, Komiya Y, Arimoto J, Horita N, Kaneko T, Iwasaki M, Nakagama H, Nakajima A (2019) Chemoprevention of colorectal cancer: past, present, and future. Cancer Sci 110(10):3018-3026. https://doi.org/10.1111/cas.14149

41. Rothwell PM, Wilson M, Elwin CE, Norrving B, Algra A, Warlow CP, Meade TW (2010) Long-term effect of aspirin on colorectal incidence and mortality: 20-year follow-up of five randomised trials. Lancet 376(9754):1741-1750. https://doi.org/10.1016/S01406736(10)61543-7

42. Bosetti C, Santucci C, Gallus S, Martinetti M, La Vecchia C (2020) Aspirin and the risk of colorectal and other digestive tract cancers: an updated meta-analysis through 2019. Ann Oncol 31(5):558-568. https://doi.org/10.1016/j.annonc.2020.02.012

43. Larsson SC, Orsini N, Wolk A (2005) Diabetes mellitus and risk of colorectal cancer: a meta-analysis. J Natl Cancer Inst 97(22):1679-1687. https://doi.org/10.1093/jnci/dji375

44. Giovannucci E (2007) Metabolic syndrome, hyperinsulinemia, and colon cancer: a review. Am J Clin Nutr 86(3):S836-S842. https://doi.org/10.1093/ajcn/86.3.836S

45. González N, Prieto I, del Puerto-Nevado L, Portal-Nuñez S, Ardura JA, Corton M, Fernández-Fernández B, Aguilera O, 
Gomez-Guerrero C, Mas S, Moreno JA, Ruiz-Ortega M, Sanz AB, Sanchez-Niño MD, Rojo F, Vivanco F, Esbrit P, Ayuso C, Alvarez-Llamas G, Egido J, García-Foncillas J, Ortiz A, DiabetesCancerConnect Consortium (2017) 2017 update on the relationship between diabetes and colorectal cancer: epidemiology, potential molecular mechanisms and therapeutic implications. Oncotarget 8(11):18456-18485. https://doi.org/10.18632/oncot arget.14472

46. Mikaeel RR, Symonds EL, Kimber J, Smith E, Horsnell M, Uylaki W, Tapia Rico G, Hewett PJ, Yong J, Tonkin D, Jesudason D, Poplawski NK, Ruszkiewicz AR, Drew PA, Hardingham JE, Wong S, Frank O, Tomita Y, Patel D, Vatandoust S, Townsend AR, Roder D, Young GP, Parry S, Tomlinson IP, Wittert G, Wattchow D, Worthley DL, Brooks WJ, Price TJ, Young JP (2020) Young-onset colorectal cancer is associated with a personal history of type 2 diabetes. Asia Pac J Clin Oncol. https:// doi.org/10.1111/ajco.13428

47. Lascar N, Brown J, Pattison H, Barnett AH, Bailey CJ, Bellary S (2018) Type 2 diabetes in adolescents and young adults. Lancet Diabetes Endocrinol 6(1):69-80. https://doi.org/10.1016/S22138587(17)30186-9

48. Cong YJ, Gan Y, Sun HL, Deng J, Cao SY, Xu X, Lu ZX (2014) Association of sedentary behaviour with colon and rectal cancer: a meta-analysis of observational studies. Br J Cancer 110(3):817826. https://doi.org/10.1038/bjc.2013.709

49. Mahmood S, MacInnis RJ, English DR, Karahalios A, Lynch BM (2017) Domain-specific physical activity and sedentary behaviour in relation to colon and rectal cancer risk: a systematic review and meta-analysis. Int J Epidemiol 46(6):1797-1813. https://doi.org/ 10.1093/ije/dyx 137

50. Namasivayam V, Lim S (2017) Recent advances in the link between physical activity, sedentary behavior, physical fitness, and colorectal cancer. F1000Res 6:199. https://doi.org/10.12688/ f1000research.9795.1

51. Berger NA (2018) Young adult cancer: influence of the obesity pandemic. Obesity (Silver Spring) 26(4):641-650. https://doi.org/ 10.1002/oby.22137

52. Dash C, Yu J, Nomura S, Lu J, Rosenberg L, Palmer JR, AdamsCampbell LL (2020) Obesity is an initiator of colon adenomas but not a promoter of colorectal cancer in the Black Women's Health Study. Cancer Causes Control 31(4):291-302. https://doi.org/10. 1007/s10552-020-01283-3

53. Bardou M, Barkun AN, Martel M (2013) Obesity and colorectal cancer. Gut 62(6):933-947. https://doi.org/10.1136/ gutjnl-2013-304701

54. Alemán JO, Eusebi LH, Ricciardiello L, Patidar K, Sanyal AJ, Holt PR (2014) Mechanisms of obesity-induced gastrointestinal neoplasia. Gastroenterology 146(2):357-373. https://doi.org/10. 1053/j.gastro.2013.11.051

55. Nimptsch K, Pischon T (2015) Body fatness, related biomarkers and cancer risk: an epidemiological perspective. Horm Mol Biol Clin Investig 22(2):39-51. https://doi.org/10.1515/ hmbci-2014-0043

56. Statovci D, Aguilera M, MacSharry J, Melgar S (2017) The impact of Western diet and nutrients on the microbiota and immune responses at mucosal interfaces. Front Immunol 8:838. https:// doi.org/10.3389/fimmu.2017.00838

57. Zinöcker MK, Lindseth IA (2018) The western-diet-microbiome-host interaction and its role in metabolic disease. Nutrients 10(3):365. https://doi.org/10.3390/nu10030365

58. Zheng X, Hur J, Nguyen LH, Liu J, Song M, Wu K, Smith-Warner SA, Ogino S, Willett WC, Chan AT, Giovannucci E, Cao Y (2020) Comprehensive assessment of diet quality and risk of precursors of early-onset colorectal cancer. J Natl Cancer Inst. https://doi.org/ 10.1093/jnci/djaa164

59. Di Rienzi SC, Britton RA (2020) Adaptation of the gut microbiota to modern dietary sugars and sweeteners. Adv Nutr 11(3):616629. https://doi.org/10.1093/advances/nmz118

60. Goncalves MD, Lu C, Tutnauer J, Hartman TE, Hwang SK, Murphy CJ, Pauli C, Morris R, Taylor S, Bosch K, Yang S, Wang Y, Van Riper J, Lekaye HC, Roper J, Kim Y, Chen Q, Gross SS, Rhee KY, Cantley LC, Yun J (2019) High-fructose corn syrup enhances intestinal tumor growth in mice. Science 363(6433):1345-1349. https://doi.org/10.1126/science.aat8515

61. Fiolet T, Srour B, Sellem L, Kesse-Guyot E, Allès B, Méjean C, Deschasaux M, Fassier P, Latino-Martel P, Beslay M, Hercberg S, Lavalette C, Monteiro CA, Julia C, Touvier M (2018) Consumption of ultra-processed foods and cancer risk: results from NutriNet-Santé prospective cohort. BMJ 360:k322. https://doi. org/10.1136/bmj.k322

62. Sylvetsky AC, Rother KI (2016) Trends in the consumption of low-calorie sweeteners. Physiol Behav 164(Pt B):446-450. https:// doi.org/10.1016/j.physbeh.2016.03.030

63. Ruiz-Ojeda FJ, Plaza-Díaz J, Sáez-Lara MJ, Gil A (2019) Effects of sweeteners on the gut microbiota: a review of experimental studies and clinical trials. Adv Nutr 10(suppl_1):S31-S48. https:// doi.org/10.1093/advances/nmy037

64. Zhao LG, Li ZY, Feng GS, Ji XW, Tan YT, Li HL, Gunter MJ, Xiang YB (2020) Coffee drinking and cancer risk: an umbrella review of meta-analyses of observational studies. BMC Cancer 20:101. https://doi.org/10.1186/s12885-020-6561-9

65. Zhu MZ, Lu DM, Ouyang J, Zhou F, Huang PF, Gu BZ, Tang JW, Shen F, Li JF, Li YL, Lin HY, Li J, Zeng X, Wu JL, Cai SX, Wang KB, Huang JA, Liu ZH (2020) Tea consumption and colorectal cancer risk: a meta-analysis of prospective cohort studies. Eur J Nutr 59(8):3603-3615. https://doi.org/10.1007/ s00394-020-02195-3

66. Keum N, Aune D, Greenwood DC, Ju W, Giovannucci EL (2014) Calcium intake and colorectal cancer risk: dose-response meta-analysis of prospective observational studies. Int J Cancer 135(8):1940-1948. https://doi.org/10.1002/ijc.28840

67. Heine-Bröring RC, Winkels RM, Renkema JM, Kragt L, van Orten-Luiten AC, Tigchelaar EF, Chan DS, Norat T, Kampman E (2015) Dietary supplement use and colorectal cancer risk: a systematic review and meta-analyses of prospective cohort studies. Int J Cancer 136(10):2388-2401. https://doi.org/10.1002/ijc. 29277

68. Zhang X, Keum N, Wu K, Smith-Warner SA, Ogino S, Chan AT, Fuchs CS, Giovannucci EL (2016) Calcium intake and colorectal cancer risk: results from the Nurses' Health Study and Health Professionals Follow-up Study. Int J Cancer 139(10):2232-2242. https://doi.org/10.1002/ijc.30293

69. Dozois EJ, Boardman LA, Suwanthanma W, Limburg PJ, Cima RR, Bakken JL, Vierkant RA, Aakre JA, Larson DW (2008) Young-onset colorectal cancer in patients with no known genetic predisposition: can we increase early recognition and improve outcome? Medicine (Baltimore) 87(5):259-263. https://doi.org/ 10.1097/MD.0b013e3181881354

70. Falony G, Joossens M, Vieira-Silva S, Wang J, Darzi Y, Faust K, Kurilshikov A, Bonder MJ, Valles-Colomer M, Vandeputte D, Tito RY, Chaffron S, Rymenans L, Verspecht C, De Sutter L, Lima-Mendez G, D'hoe K, Jonckheere K, Homola D, Garcia R, Tigchelaar EF, Eeckhaudt L, Fu J, Henckaerts L, Zhernakova A, Wijmenga C, Raes J (2016) Population-level analysis of gut microbiome variation. Science 352(6285):560-564. https://doi. org/10.1126/science.aad3503 
71. Francino MP (2016) Antibiotics and the human gut microbiome: dysbioses and accumulation of resistances. Front Microbiol 6:1543. https://doi.org/10.3389/fmicb.2015.01543

72. Zhu Q, Gao R, Wu W, Qin H (2013) The role of gut microbiota in the pathogenesis of colorectal cancer. Tumour Biol 34(3):12851300. https://doi.org/10.1007/s13277-013-0684-4

73. Zhang J, Haines C, Watson AJM, Hart AR, Platt MJ, Pardoll DM, Cosgrove SE, Gebo KA, Sears CL (2019) Oral antibiotic use and risk of colorectal cancer in the United Kingdom, 1989-2012: a matched case-control study. Gut 68(11):1971-1978. https://doi. org/10.1136/gutjnl-2019-318593

74. Armstrong D, Dregan A, Ashworth M, White P, McGee C, de Lusignan S (2020) The association between colorectal cancer and prior antibiotic prescriptions: case control study. Br J Cancer 122(6):912-917. https://doi.org/10.1038/s41416-019-0701-5

75. Qu G, Sun C, Sharma M, Uy JP, Song EJ, Bhan C, Shu L (2020) Is antibiotics use really associated with increased risk of colorectal cancer? An updated systematic review and meta-analysis of observational studies. Int J Colorectal Dis 35(8):1397-1412. https://doi.org/10.1007/s00384-020-03658-z

76. Simin J, Fornes R, Liu Q, Olsen RS, Callens S, Engstrand L, Brusselaers N (2020) Antibiotic use and risk of colorectal cancer: a systematic review and dose-response meta-analysis. Br J Cancer 123(12):1825-1832. https://doi.org/10.1038/s41416-020-01082-2

77. Imperiale TF, Kahi CJ, Stuart JS, Qi R, Born LJ, Glowinski EA, Rex DK (2008) Risk factors for advanced sporadic colorectal neoplasia in persons younger than age 50. Cancer Detect Prev 32(1):33-38. https://doi.org/10.1016/j.cdp.2008.01.003

78. Mathews JD, Forsythe AV, Brady Z, Butler MW, Goergen SK, Byrnes GB, Giles GG, Wallace AB, Anderson PR, Guiver TA, McGale P, Cain TM, Dowty JG, Bickerstaffe AC, Darby SC (2013) Cancer risk in 680,000 people exposed to computed tomography scans in childhood or adolescence: data linkage study of 11 million Australians. BMJ 346:f2360. https://doi.org/ 10.1136/bmj.f2360

79. Brenner DJ, Doll R, Goodhead DT, Hall EJ, Land CE, Little JB, Lubin JH, Preston DL, Preston RJ, Puskin JS, Ron E, Sachs RK, Samet JM, Setlow RB, Zaider M (2003) Cancer risks attributable to low doses of ionizing radiation: assessing what we really know. Proc Natl Acad Sci USA 100(24):13761-13766. https://doi.org/ 10.1073/pnas.2235592100

80. Vaiserman A, Koliada A, Zabuga O, Socol Y (2018) Health impacts of low-dose ionizing radiation: current scientific debates and regulatory issues. Dose Response 16(3):1559325818796331. https://doi.org/10.1177/1559325818796331

81. Fereidouni M, Ferns GA, Bahrami A (2020) Current status and perspectives regarding the association between allergic disorders and cancer. IUBMB Life 72(7):1322-1339. https://doi.org/10. 1002/iub. 2285

82. Jacobs EJ, Gapstur SM, Newton CC, Turner MC, Campbell PT (2013) Hay fever and asthma as markers of atopic immune response and risk of colorectal cancer in three large cohort studies.
Cancer Epidemiol Biomark Prev 22(4):661-669. https://doi.org/ 10.1158/1055-9965.EPI-12-1229

83. Ye J, Talaiti A, Ma Y, Zhang Q, Ma L, Zheng H (2017) Allergies and risk of colorectal cancer: a systematic review and metaanalysis of observational studies. Oncotarget 8(9):14646-14654. https://doi.org/10.18632/oncotarget.14599

84. Ma W, Yang J, Li P, Lu X, Cai J (2017) Association between allergic conditions and colorectal cancer risk/mortality: a metaanalysis of prospective studies. Sci Rep 7(1):5589. https://doi.org/ 10.1038/s41598-017-04772-9

85. Jensen-Jarolim E, Bax HJ, Bianchini R, Capron M, Corrigan C, Castells M, Dombrowicz D, Daniels-Wells TR, Fazekas J, Fiebiger E, Gatault S, Gould HJ, Janda J, Josephs DH, Karagiannis P, Levi-Schaffer F, Meshcheryakova A, Mechtcheriakova D, Mekori Y, Mungenast F, Nigro EA, Penichet ML, Redegeld F, Saul L, Singer J, Spicer JF, Siccardi AG, Spillner E, Turner MC, Untersmayr E, Vangelista L, Karagiannis SN (2017) AllergoOncologythe impact of allergy in oncology: EAACI position paper. Allergy 72(6):866-887. https://doi.org/10.1111/all.13119

86. Rennert G (2017) Reproductive factors, hormones and colorectal cancer-still unresolved. Br J Cancer 116(1):1-3. https://doi.org/ $10.1038 /$ bjc. 2016.388

87. Guan HB, Wu QJ, Gong TT, Lin B, Wang YL, Liu CX (2013) Parity and risk of colorectal cancer: a dose-response meta-analysis of prospective studies. PLoS ONE 8(9):e75279. https://doi.org/10. 1371/journal.pone.0075279

88. Merrill RM, Fugal S, Novilla LB, Raphael MC (2005) Cancer risk associated with early and late maternal age at first birth. Gynecol Oncol 96(3):583-593. https://doi.org/10.1016/j.ygyno.2004.11. 038

89. Bosetti C, Bravi F, Negri E, La Vecchia C (2009) Oral contraceptives and colorectal cancer risk: a systematic review and metaanalysis. Hum Reprod Update 15(5):489-498. https://doi.org/10. 1093/humupd/dmp017

90. Lin KJ, Cheung WY, Lai JY, Giovannucci EL (2012) The effect of estrogen vs. combined estrogen-progestogen therapy on the risk of colorectal cancer. Int J Cancer 130(2):419-430. https://doi.org/ 10.1002/ijc. 26026

91. Wernli KJ, Wang Y, Zheng Y, Potter JD, Newcomb PA (2009) The relationship between gravidity and parity and colorectal cancer risk. J Womens Health (Larchmt) 18(7):995-1001. https://doi.org/ 10.1089/jwh.2008.1068

92. Statistics Canada. Table 13-10-0096-01 Health characteristics, annual estimates. https://doi.org/10.25318/1310009601-eng. Accessed 2 Aug 2020

Publisher's Note Springer Nature remains neutral with regard to jurisdictional claims in published maps and institutional affiliations. 